\title{
Senescence of multicellular individuals: imbalance of epigenetic and non-epigenetic information in histone modifications
}

\author{
Felipe A. Veloso (D) * \\ Facultad de Ciencias, Universidad Mayor, Santiago, Chile.
}

(Dated: April 27, 2018)

\begin{abstract}
Cellular aging has been progressively elucidated by science. However, aging at the multicellular-individual level is still poorly understood. A recent theory of individuated multicellularity describes the emergence of crucial information content for cell differentiation. This information is mostly conveyed in the non-epigenetic constraints on histone modifications near transcription start sites. According to this theory, the non-epigenetic content emerges at the expense of the information capacity for epigenetic content. However, it is unclear whether this "reassignment" of capacity continues after adulthood. To answer this question, I analyzed publicly available high-throughput data of histone $\mathrm{H} 3$ modifications and mRNA abundance in human primary cells. The results show that the "reassignment" continues after adulthood in humans. Based on this evidence, I present a falsifiable theory describing how continued "reassignment" of information capacity creates a growing epigenetic/non-epigenetic information imbalance. According to my theoretical account, this imbalance is the fundamental reason why individuated multicellular organisms senesce.
\end{abstract}

Keywords: aging; ageing; cancer; constraints; naked mole-rat; bristlecone pine; Turritopsis; gene regulation; epigenetics; teleodynamics

13 Our intellectual endeavors have entertained the 14 prospect of unlimited lifespan for centuries [1], and the 15 scientific endeavor has been no exception [2]. In the 1950s, 16 the immortality of cultured somatic cells was indeed a 17 widely-held belief [3]. That changed only when Hayflick 18 \& Moorhead showed that cultured human somatic cells ${ }_{19}$ do stop dividing and become less viable once their 20 divisions reach a certain number [4], a phenomenon known 21 today as the Hayflick limit [3]. This loss of replicative ${ }_{22}$ capacity and, in general, the process of aging at the 23 cellular level, have been found to correlate with telomere 24 length $[3,5,6]$. Yet, the number of times human cells can 25 divide in culture exceeds the number of times cells divide 26 throughout our lifespan; there is no significant correlation ${ }_{27}$ between human cell replicative capacity and cell donor ${ }_{28}$ age [7]. That is, we and individuated multicellular 29 organisms in general - age before most of our cells do [8,9]. so The outstanding question is why.

31 Theoretical descriptions of senescence or aging at the 32 multicellular-individual level have been classified into 33 two categories: programmed senescence and senescence 34 caused by damage/error [10]. Recently it has been 35 argued, however, that senescence is not programmed 36 nor is it ultimately a consequence of damage or error 37 in the organism's structure/dynamics [11]. Instead, 38 it may be a byproduct of maintenance and/or 39 developmental dynamics [11,12], themselves underpinned 40 in part by intracellular signaling pathways such as 41 the cell-cycle-related PI3K/AKT/mTOR pathway [11]. ${ }_{42}$ These pathways have been shown to modulate aging 43 at the cellular level in species such as the yeast 44 Saccharomyces cerevisiae [13].

45 The analogous notion of aging at the 46 multicellular-individual level as a byproduct of

* Correspondence: veloso.felipe.a@gmail.com
47 certain functional signaling pathways [11] is, in principle, 48 supported by the fact that the deficiency of mTOR 49 kinase - a key component of the PI3K/AKT/mTOR 50 pathway - can double the lifespan of the roundworm ${ }_{51}$ Caenorhabditis elegans [14]. However, the fundamental 52 dynamics that make individuated multicellular organisms 53 senescent after adulthood remain unclear and largely ${ }_{54}$ lack falsifiable scientific theories. Falsifiability-the 55 possibility of establishing a hypothesis or theory as ${ }_{56}$ false by observation and experiment [15] - allows the 57 objective rejection of existing scientific theories, fosters ${ }_{58}$ the development of new ones, and constitutes the 59 most widely accepted demarcation between science 60 and non-science [16].

${ }_{61}$ Using publicly available high-throughput data of 62 histone $\mathrm{H} 3$ modifications and mRNA abundance in human 63 primary cells to look for proof of concept, the issue of 64 senescence can also be approached from the angle of 65 theoretical biology. Thus, I conducted a statistical data ${ }_{66}$ analysis in this study, which revealed that proof of concept 67 exists for the human species. These findings provide ${ }_{68}$ empirical grounds for my theoretical work, suggesting that 69 senescence is a byproduct of functional developmental 70 dynamics as first described by a recently proposed 71 theory of individuated multicellularity [17]. Specifically, 72 I show that the byproduct is a post-ontogenetic, 73 growing imbalance between two different information 74 contents conveyed respectively in two different types of 75 constraints on histone post-translational modifications 76 near transcription start sites (TSSs). Constraints are 77 here understood as the local and level-of-scale specific 78 thermodynamic boundary conditions required for energy 79 to be released as work as described by Atkins [18]. ${ }_{80}$ The concept of constraint is crucial because, according to 81 the theory of individuated multicellularity, a higher-order 82 constraint (i.e., a constraint on constraints) on changes 83 in histone modifications harnesses critical work that 
${ }_{84}$ regulates transcriptional changes for cell differentiation 85 at the multicellular-individual level.

${ }_{86}$ Under the theory of individuated multicellularity, 105 as changes in gene expression that cannot be explained 87 the intrinsic higher-order constraint is the simplest 106 by (i.e., that are explicitly unrelated to) changes in the ${ }_{88}$ multicellular individual in fundamental terms. In addition, ${ }_{107}$ DNA sequence [22]. This relevance is underpinned by the 89 the dynamics of the lower-order constraints must be 108 capacity of histone modifications to convey information 90 explicitly unrelated to each other (i.e., statistically 109 content, which has allowed the prediction of mRNA ${ }_{91}$ independent) in order to elicit the emergence of ${ }_{110}$ levels from histone modification profiles near TSSs with 92 the intrinsic higher-order constraint. Along with the 111 high accuracy [23].

${ }_{93}$ emergence of this intrinsic higher-order constraint, the ${ }_{112}$ Based on these considerations and the properties of the 94 theory of individuated multicellularity describes the 113 nonnegative measure of multivariate statistical association ${ }_{95}$ emergence of critical information content, named in the ${ }_{114}$ known as total correlation [24] or multiinformation [25] 96 theory hologenic content, which is about the multicellular ${ }_{115}$ (symbolized by $C$ and typically measured in bits), the ${ }_{97}$ individual as a whole in terms of developmental ${ }_{116}$ overall observable histone crosstalk can be decomposed. 98 self-regulation. Thus, for the sake of brevity, I here refer ${ }_{117}$ That is, histone crosstalk, if measured as a total 99 to the theory of individuated multicellularity as the ${ }_{118}$ correlation $C$, is the sum of two explicitly unrelated 100 hologenic theory.

${ }_{119} C$ components: one epigenetic (i.e., explicitly related to 101 The constraints on the combinatorial patterns of 120 changes in gene expression) and the other non-epigenetic 102 histone modifications are generally known as histone ${ }_{121}$ (i.e., explicitly unrelated to changes in gene expression). ${ }_{122}$ This sum can be expressed as follows:
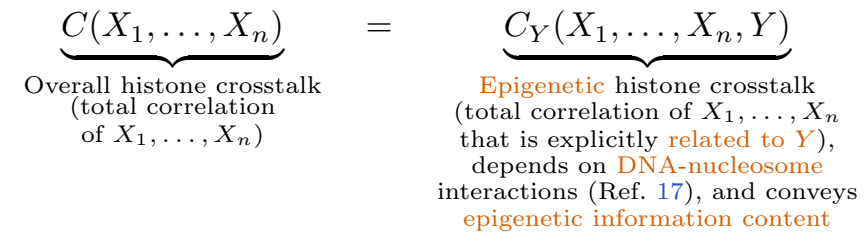

123 124 histone modification levels in specific genomic positions 125 with respect to the TSS and $Y$ is a random variable ${ }_{126}$ representing either gene expression level, transcription ${ }_{127}$ rate, or mRNA abundance level associated with the TSS. ${ }_{128}$ These levels are equivalent for the decomposition because 129 of the strong correlation that exists between them ([26] 130 and references therein).

${ }_{131}$ The hologenic theory describes how the epigenetic ${ }_{132}$ component of histone crosstalk (represented by ${ }_{133} C_{Y}\left(X_{1}, \ldots, X_{n}, Y\right)$ in the sum decomposition of Eq. 1) 134 conveys information about each cell's transcriptional 1 135 profile. This component is, in information content 1 136 terms, the dominating component for any eukaryotic ${ }_{137}$ colonial species (such as the alga Volvox carteri [27]) and, 138 importantly, also for undifferentiated stem cells.

139 The second, non-epigenetic component of histone 140 crosstalk (represented by $C\left(X_{1}, \ldots, X_{n} \mid Y\right)$ in Eq. 1) is 141 known to grow in magnitude during development until the 142 organism's mature form is reached [17]. This component 143 is described by the hologenic theory as conveying 144 information about the multicellular individual as a 171 145 whole - starting from the moment said individual emerges ${ }_{146}$ as an intrinsic higher-order constraint on the early ${ }_{147}$ embryo's proliferating cells.

${ }_{148}$ Importantly, the overall observable histone crosstalk 149 magnitude (represented by $C\left(X_{1}, \ldots, X_{n}\right)$ in Eq. 1) is
150 not infinite. In other words, the overall histone crosstalk ${ }_{151}$ has a finite information capacity, which can be measured 152 in bits. Moreover, the sum decomposition in Eq. 1 implies 153 that the growth in magnitude (bits) of the hologenic 154 (i.e., non-epigenetic) component must be accompanied 155 by a decrease in magnitude of the epigenetic component. 156 That is, the capacity (in bits) for hologenic information 157 content in histone crosstalk is bound to grow at the 158 expense of the capacity for epigenetic information content. 159 The hologenic theory also maintains that a necessary 160 condition for the evolution of individuated multicellular 161 lineages was the appearance of a class of molecules 162 synthesized by the cells - called Nanney's extracellular ${ }_{163}$ propagators (symbolized by $F_{N}$ ) in the theory [17]. ${ }_{164}$ These $F_{N}$ molecules are predicted to be, in a given 165 tissue and time period, (i) secretable into the extracellular 166 space, (ii) once secreted, capable of eliciting a significant 167 incremental change (via signal transduction) in the 168 magnitude of the non-epigenetic histone crosstalk (i.e., the ${ }_{169} C\left(X_{1}, \ldots, X_{n} \mid Y\right)$ summand in Eq. 1) within other 170 cells' nuclei, and (iii) affected in their extracellular 171 diffusion dynamics by the geometrical complexity of the 172 extracellular space (i.e., constraints on diffusion at the 173 multicellular-individual level, which cannot be reduced to 174 constraints at the cellular level). Also under the hologenic 175 theory, for the multicellular individual to develop and 176 survive, both hologenic (developmental self-regulation of 
bioRxiv preprint doi: https://doi.org/10.1101/310300; this version posted April 28, 2018. The copyright holder for this preprint (which was not certified by peer review) is the author/funder, who has granted bioRxiv a license to display the preprint in perpetuity. It is made available under aCC-BY 4.0 International license.

177 the multicellular individual overall) and epigenetic (each 178 cell's transcriptional profile) contents must coexist.

179 One final but important consideration regarding 180 histone crosstalk is that it is the result of constraints 198 a significant loss of hologenic content in cancer cells, 181 which, as mentioned previously, are level-of-scale specific. 199 because they are no longer constrained by the multicellular ${ }_{182}$ To exemplify this specificity, consider the example of an 200 individual that normal (i.e., non-cancerous) cells serve and ${ }_{183}$ internal combustion engine: a single molecule in a cylinder 201 are constrained by. Thus, I developed a falsifiable theory 184 wall does not embody a constraint on the expansion 202 of senescence based on the post-ontogenetic continuation 185 of the igniting gas, yet the cylinder-piston ensemble ${ }_{203}$ of this "reassignment" process in human histone crosstalk 186 does. For this reason, histone crosstalk constraints were 204 as proof of concept.

${ }_{187}$ expected to have relevance for senescence but only at a ${ }_{205}$ To test this theory, I formalized the proof of concept 188 specific level of scale. The specific level of scale in histone 206 into the following two hypotheses: (i) within genomic 189 crosstalk that is relevant for human senescence has not ${ }_{207}$ regions adjacent to TSSs in primary normal cells, the 190 been studied in detail before.

208 log-ratio between the non-epigenetic and epigenetic ${ }_{191}$ To investigate from a theoretical angle if the 209 histone H3 crosstalk magnitudes is significantly and 192 "reassignment" of information capacity for epigenetic 210 positively correlated with cell donor age (over a range of 193 and non-epigenetic (i.e., hologenic) content stops when 211 0-90 years old) and (ii) no such statistically significant 194 development reaches the multicellular individual's mature ${ }_{212}$ correlation exists for primary cancer cells (see Fig. 1).

a
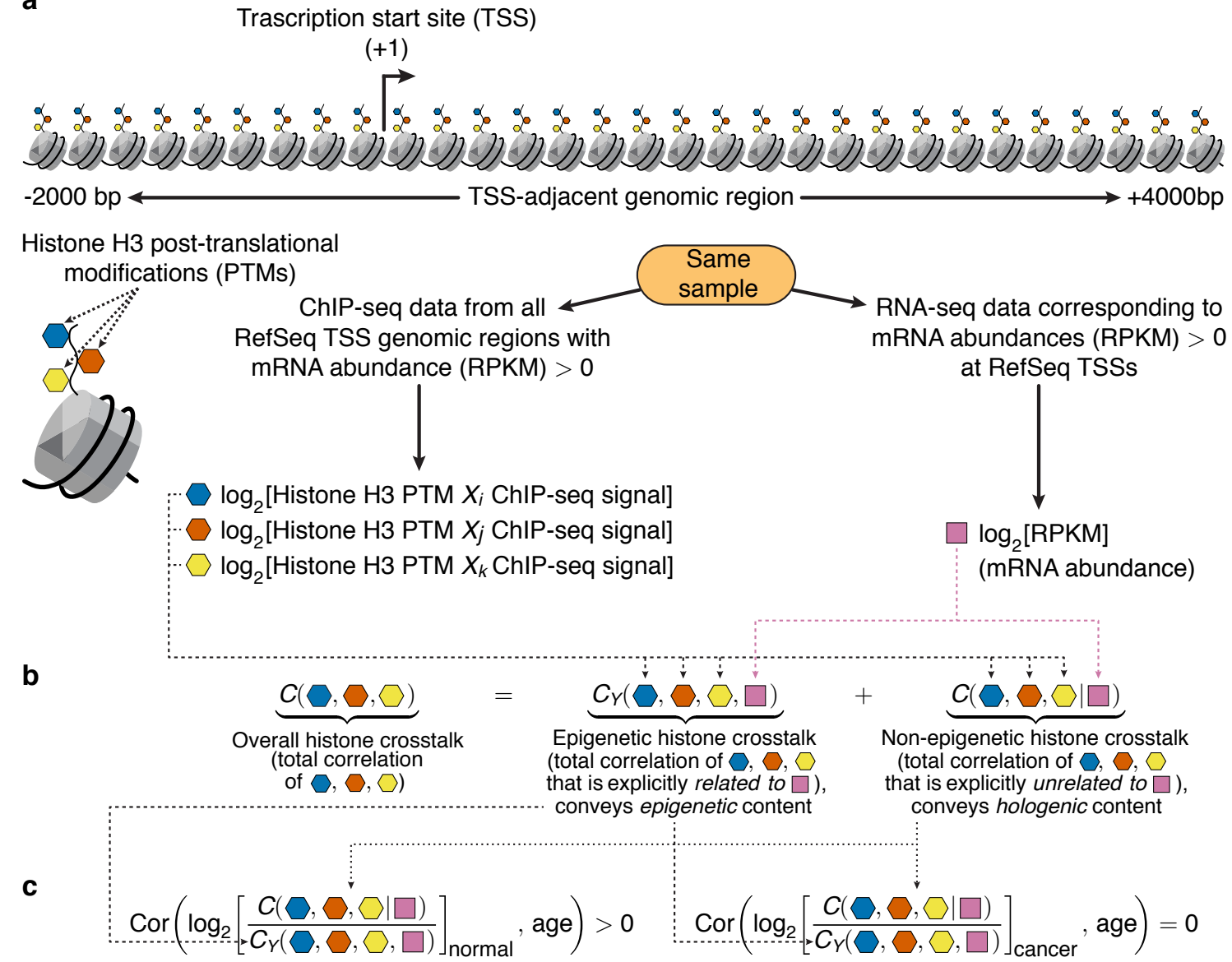

Fig. 1. Schematic for proof-of-concept hypotheses and computational analysis for testing. Publicly available ChIP-seq (chromatin immunoprecipitation followed by high-throughput DNA sequencing) and RNA-seq (transcriptome high-throughput sequencing) data for human primary cell samples allowed the computation, for each TSS, of position-specific histone H3 modification levels (at every 200bp) and its associated mRNA abundance level (a). After log-transforming these levels and taking into account all TSSs, the TSS-adjacent histone H3 crosstalk (triad-wise crosstalk depicted here) was represented as a total correlation [24] or information capacity in bits, which in turn was decomposed as the sum of two measurable and explicitly unrelated components: one epigenetic (explicitly related to transcriptional changes) and the other non-epigenetic (explicitly unrelated to said changes) (b). Taking into account all samples, the log-ratio of non-epigenetic to epigenetic histone H3 crosstalk magnitude was hypothesized to be positively correlated with cell donor age in normal cells (c, left) and also to be uncorrelated with cell donor age in cancer cells (c, right). The subsequent rejection of the statistical null hypothesis in (c, left) and the failure to reject the statistical null hypothesis in (c, right) provided proof of concept for the theory of senescence proposed in this paper. 


\section{RESULTS}

213 To test the two proof-of-concept hypotheses, ${ }_{214}$ I used publicly available ChIP-seq (chromatin 215 immunoprecipitation followed by high-throughput ${ }_{216}$ DNA sequencing) and RNA-seq (transcriptome 217 high-throughput sequencing) data for human primary cell 218 samples, which were obtained from different individuals 219 with ages ranging from 0 to +90 years old. I computed 220 log-transformed ChIP-seq signal magnitudes for each 221 primary cell sample from ChIP-seq data of different 222 position-specific (in bp relative to the TSS) histone H3 223 modifications. Similarly, I log-transformed mRNA 224 abundance values from RNA-seq assay data associated 225 with each ChIP-seq assay for each primary cell sample.

${ }_{226}$ Using these tandem ChIP-seq and RNA-seq data, ${ }_{227} \mathrm{I}$ quantified the non-epigenetic and epigenetic 228 histone H3 crosstalk magnitudes (Eq. 1) for triads 229 of variables $\left\{X_{i}, X_{j}, X_{k}\right\}$. These variables represented 230 position-specific histone H3 modification levels, 231 i.e., $C\left(X_{i}, X_{j}, X_{k} \mid Y\right)$ and $C_{Y}\left(X_{i}, X_{j}, X_{k}, Y\right)$ for 232 the non-epigenetic and epigenetic histone crosstalk ${ }_{233}$ components, respectively, where $Y$ represents mRNA ${ }_{234}$ abundance. Triads (as opposed to pairs or tetrads) were 235 first analyzed because a triad constitutes the number 236 of variables (i.e., position-specific histone modification ${ }_{237}$ levels) found to possess both significant predictive ${ }_{238}$ power and predictive synergy to resolve the statistical 239 uncertainty about the mRNA abundance level associated ${ }_{240}$ with a given TSS (see details in Methods).

241 The log-ratio (base 2) between the non-epigenetic and 242 epigenetic histone H3 crosstalk magnitudes was thus 243 computed as the dimensionless quantity

$$
\log _{2}\left[\frac{C\left(X_{i}, X_{j}, X_{k} \mid Y\right)}{C_{Y}\left(X_{i}, X_{j}, X_{k}, Y\right)}\right] .
$$

244 Importantly, total correlation $C$ captures all possible 245 associations in the set of variables $\left\{X_{i}, X_{j}, X_{k}\right\}$ that may 246 exist starting from the pairwise level.

\section{The log-ratio of non-epigenetic to epigenetic histone $\mathrm{H} 3$ crosstalk magnitude is positively correlated with cell donor age in normal cells}

247 ChIP-seq data for five histone H3 modifications 248 were used in all analyses: H3K4me1 (histone H3 249 lysine 4 monomethylation), H3K9me3 (histone H3 250 lysine 9 trimethylation), H3K27ac (histone H3 251 lysine 27 acetylation), H3K27me3 (histone H3 lysine 27 252 trimethylation), and H3K36me3 (histone H3 lysine 36 253 trimethylation). The ChIP-seq signals for these 254 modifications were computed for $30200 \mathrm{bp}$-long genomic 255 bins across a $6,000 \mathrm{bp}$-long TSS-adjacent region (see ${ }_{256}$ Fig. 1). Thus, a total of 150 variables $X_{i}$ representing

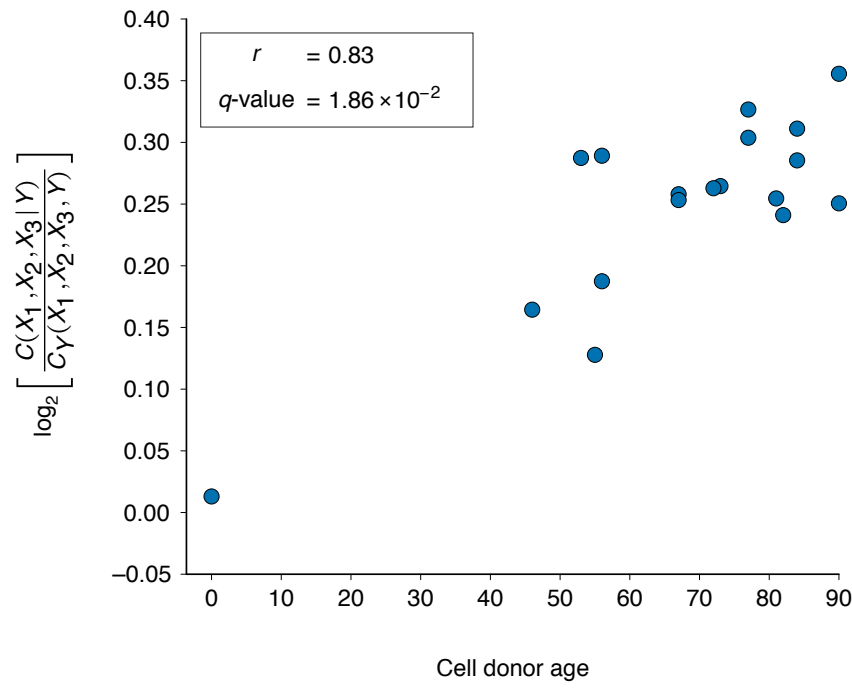

Fig. 2. Positive correlation between the log-ratio of non-epigenetic to epigenetic histone $\mathrm{H3}$ crosstalk magnitude and cell donor age for one triad of position-specific histone $\mathrm{H3}$ modification levels in normal cells. In this triad $X_{1}$ represents H3K27ac (at $-1000 \mathrm{bp}), X_{2}$ represents H3K36me3 (at $\left.+1000 \mathrm{bp}\right)$, and $X_{3}$ represents $\mathrm{H} 3 \mathrm{~K} 4 \mathrm{me1}$ (at $+3200 \mathrm{bp}$ ), which together comprise all TSSs. Regardless of the specific triad, $Y$ always represents the mRNA abundance profile comprising all TSSs. Each data point in the figure corresponds to a primary cell sample.

257 position-specific histone H3 modification levels-each 258 variable with signals for 18,220 RefSeq TSSs - were used 259 when analyzing each cell sample. A total of 18 normal 260 cell samples and 17 cancer cell samples was included 261 in the analysis. The Pearson correlation coefficient $r$ 262 between the log-ratio and the cell donor age was obtained 263 for each of the $\left(\begin{array}{c}150 \\ 3\end{array}\right)=551,300$ possible $\left\{X_{i}, X_{j}, X_{k}\right\}$ 264 triads. The 551,300 $p$-values (one-sided Student's $t$-test) 265 associated to these $r$ values were then corrected for 266 multiple testing (Benjamini-Yekutieli correction, see ${ }_{267}$ Methods), obtaining $q$-values.

${ }_{268}$ To determine whether the hypothesized positive 269 correlation between the non-epigenetic/epigenetic 270 histone $\mathrm{H} 3$ crosstalk log-ratio and cell donor age exists, 271 and also to illustrate the concept of positive correlation in 272 normal cells vs. no correlation cancer cells, I obtained all 273 possible 551,300 correlation values for triads (one-sided 274 Student's t-test). To exemplify, the results for the triad 275 \{H3K27ac (at -1000bp), H3K36me3 (at +1000bp), ${ }_{276} \mathrm{H} 3 \mathrm{~K} 4 \mathrm{me1}$ (at $\left.\left.+3200 \mathrm{bp}\right)\right\}$ are shown here, where the 277 correlation was positive $(r=0.83)$ and highly significant $278\left(q=1.86 \times 10^{-2}\right)$, as seen in Fig. 2, indicating that the 279 hypothesized correlation holds for this triad.

${ }_{280}$ Altogether, the 551,300 correlation values had a mean 281 value $\bar{r}=0.58$, a median value $\tilde{r}=0.67$, and a standard 282 deviation value $\sigma_{r}=0.24$ (see statistical distribution 283 of $r$ in Fig. 3). From these correlation values, only 28424,185 (i.e., $\sim 4 \%$ ) were nonpositive and none of them 285 was statistically significant (i.e., where $r \leq 0, q>0.05$ ). ${ }_{286}$ In contrast, it was found that for 315,378 triads 
bioRxiv preprint doi: https://doi.org/10.1101/310300; this version posted April 28, 2018. The copyright holder for this preprint (which was not certified by peer review) is the author/funder, who has granted bioRxiv a license to display the preprint in perpetuity. It is made available under aCC-BY 4.0 International license.

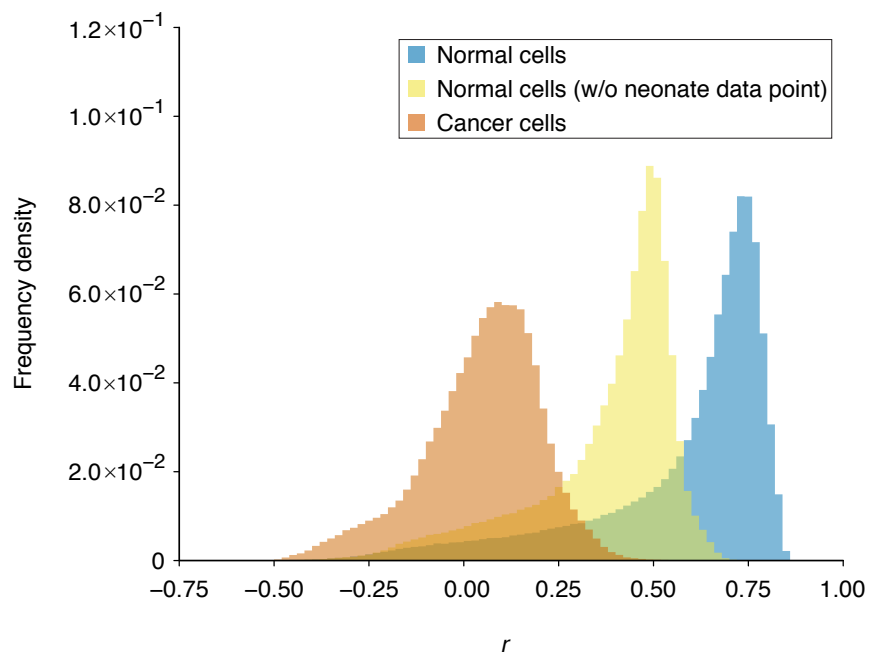

Fig. 3. Statistical distribution of the correlation coefficient $(r)$ between the log-ratio of non-epigenetic to epigenetic histone $\mathrm{H} 3$ crosstalk magnitude (triad-wise) and cell donor age. Histograms represent $r$ values for all 551,300 possible triads in normal cells (blue), normal cells excluding the neonate data point (yellow), and cancer cells (orange).

287 (i.e., $\sim 57 \%$ ) the correlation values were positive and 288 statistically significant (i.e., $r>0$ and $q \leq 0.05$ ).

289 Importantly, I also found that the hypothesized positive 290 correlation between the log-ratio of non-epigenetic to 291 epigenetic histone $\mathrm{H} 3$ crosstalk and cell donor age verified 292 for triads of position-specific histone H3 modifications 293 in normal cells loses its strength for tetrads $(\bar{r}=0.34$; $\left.294 \tilde{r}=0.35 ; \sigma_{r}=0.27\right)$. It is also no longer greater than 295 zero for pairs $\left(\bar{r}=-0.30 ; \tilde{r}=-0.38 ; \sigma_{r}=0.40\right)$ (Fig. 4). 296 These results for tetrads and pairs indicate that the 297 predicted positive correlation only holds for triads ${ }_{298}$ (and it was predicted in the second proof-of-principle 299 hypothesis not to hold in cancer cells). Such specificity 300 was expected because if senescence can be explained 301 in terms of an imbalance of information-conveying 302 constraints that are level-of-scale specific like other 303 thermodynamic constraints, the imbalance itself also must 304 be level-of-scale specific.

The log-ratio of non-epigenetic to epigenetic histone $\mathrm{H3}$ crosstalk magnitude does not correlate with cell donor age in cancer cells

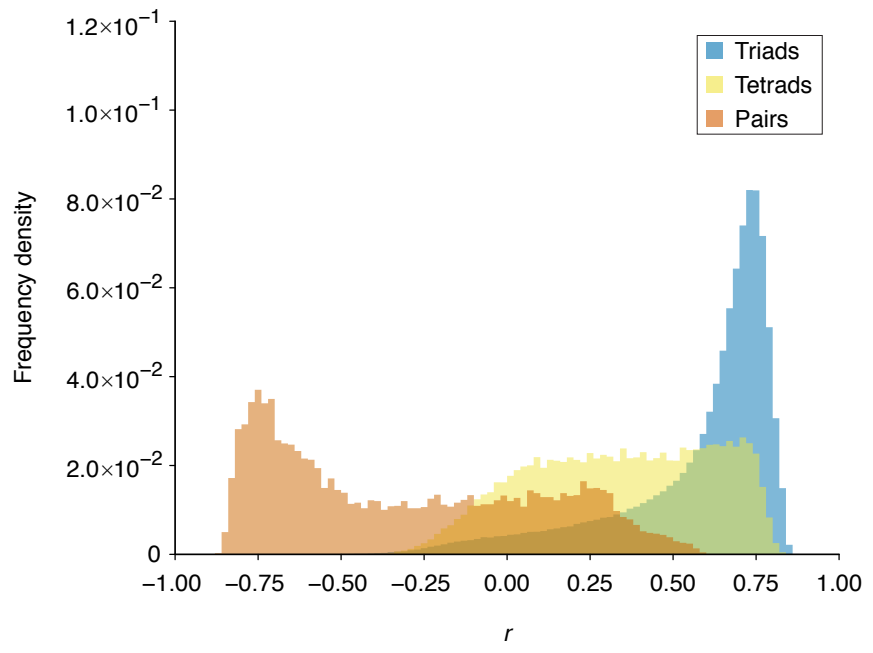

Fig. 4. Statistical distribution of the correlation coefficient $(r)$ between the log-ratio of non-epigenetic to epigenetic histone H3 crosstalk magnitude and cell donor age for triads, tetrads, and pairs of position-specific histone $\mathrm{H} 3$ modification levels. Histograms represent $r$ values for all 551,300 possible triads (blue), 50,000 random tetrads (yellow), and all 11,175 possible pairs (orange).

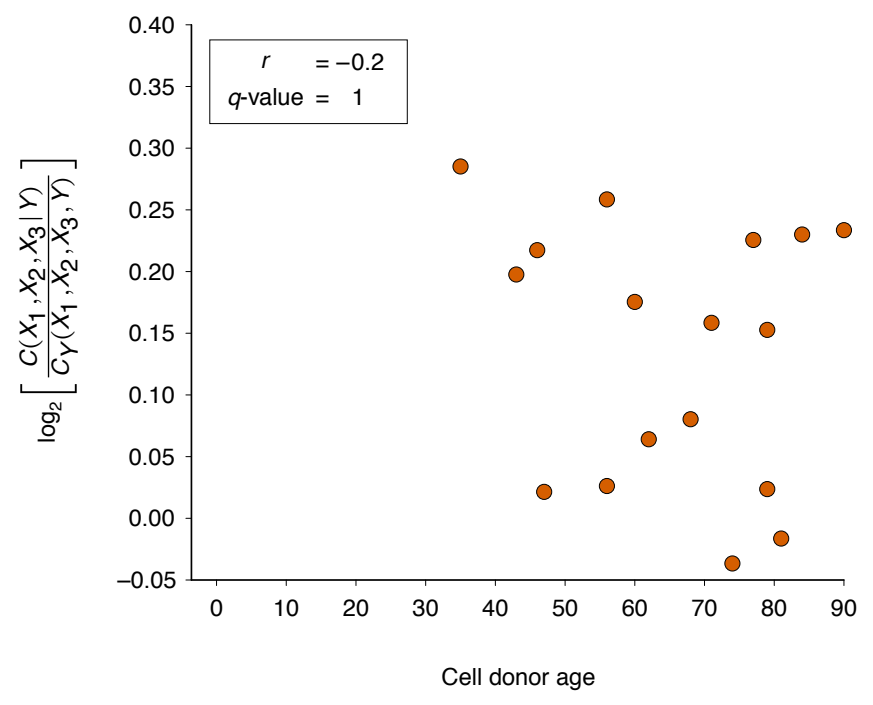

Fig. 5. No significant correlation between the log-ratio of non-epigenetic to epigenetic histone $\mathrm{H3}$ crosstalk magnitude and cell donor age for one triad of position-specific histone $\mathrm{H3}$ modification levels in cancer cells. This is the same triad of Fig. 2, i.e., $X_{1}$ representing $\mathrm{H} 3 \mathrm{~K} 27 \mathrm{ac}$ (at $-1000 \mathrm{bp}$ ), $X_{2}$ representing H3K36me3 (at $+1000 \mathrm{bp}$ ), and $X_{3}$ representing H3K4me1 (at $+3200 \mathrm{bp}$ ).

305 When I analyzed the log-ratio of non-epigenetic to $316 \sigma_{r}=0.15$ (see statistical distribution of $r$ in Fig. 3). 306 epigenetic histone $\mathrm{H} 3$ crosstalk magnitude and cell 317 All associated $p$-values (two-sided Student's $t$-test) were 307 donor age for cancer cells using the same exemplary 318 corrected and the resulting $q$-values were all equal to 308 triad \{H3K27ac (at -1000bp), H3K36me3 (at $+1000 \mathrm{bp}$ ), 3191 and hence non-significant. Similar results-i.e., all 309 H3K4me1 (at $+3200 \mathrm{bp})\}$, I found that no significant $320 q$-values equal to 1 -were obtained for all 11,175 pairs of 310 correlation exists between those two variables $(r=-0.2 ; 321$ position-specific histone H3 modification levels $(\bar{r}=-0.04$; $311 q=1$; see Fig. 5), as hypothesized.

$\left.322 \tilde{r}=-0.05 ; \sigma_{r}=0.23\right)$ and for all 50,000 random tetrads 312 For the 551,300 correlation values corresponding to ${ }_{323}\left(\bar{r}=0.08 ; \tilde{r}=0.11 ; \sigma_{r}=0.15\right)$. These results suggest that, 313 all triads of position-specific histone H3 modifications 324 as predicted, no significant correlation exists between 314 in cancer cells, the mean and median were close to 325 the log-ratio of non-epigenetic to epigenetic histone $\mathrm{H} 3$ 315 zero $(\bar{r}=0.05 ; \tilde{r}=0.07)$, and the standard deviation was 326 crosstalk magnitude and cell donor age in cancer cells. 
${ }_{327}$ I also evaluated whether the stark difference of the ${ }_{377}$ respectively. These results suggest that senescence 328 correlation values between normal-i.e., $r$ markedly 378 would indeed be an information capacity "reassignment" 329 positive - and cancer cells - i.e., $r$ close to zero - was only 379 problem - creating in turn an information-content 330 attributable to the data point (for normal cell samples) 380 imbalance, as hypothesized - rather than a "total capacity 331 that corresponds to a neonate, with coordinates $(0,0.01)$ 381 contraction" problem.

332 in Fig. 2. In other words, whether the neonate data point ${ }_{382}$ Taken together, the statistical strength of all the results 333 was simply a statistical outlier that created an otherwise 383 obtained-notwithstanding the heterogeneous origin of 334 nonexistent difference between normal and cancer cells in 384 the primary cell samples analyzed given the different 335 the analysis.

336 For this purpose, I recomputed all 551,300 correlation 337 values corresponding to normal cells, excluding the 338 neonate data point. The mean, median, and standard 339 deviation values obtained were $\bar{r}=0.38, \tilde{r}=0.44$, and $340 \sigma_{r}=0.19$, respectively (see distribution of $r$ in comparison 341 with that for cancer cells in Fig. 3). This difference 342 between $r$ values for normal cells (neonate data point 343 excluded) and cancer cells was further tested and shown to 344 be highly significant (Mann-Whitney $U$ test: $U=2.7 \times 10^{11}$, $\left.{ }_{345} p<2.2 \times 10^{-16}\right)$. These findings suggest that the neonate 346 data point is not a statistical outlier among normal 347 cell samples let alone explains the difference between 348 normal and cancer cells in terms of the correlation 349 values obtained.

\section{The total information capacity of triad-wise histone $\mathrm{H} 3$ crosstalk does not correlate with cell donor age} 385 tissues from different individuals - provides proof of 386 concept and underpins a strong falsifiable prediction for 387 a theory of senescence presented in the Discussion.

\section{DISCUSSION}

${ }_{388}$ The successful testing of the two proof-of-concept 389 hypotheses in the present work provides empirical grounds 390 for the following falsifiable theory of senescence as a 391 byproduct of developmental dynamics: Given that the 392 "reassignment" process for information capacity in histone ${ }_{393}$ crosstalk -i.e., a progressive gain of capacity for hologenic 394 information content at the expense of that for epigenetic 395 content-continues without interruption throughout 396 the multicellular individual's lifespan, a growing and 397 ultimately lethal information content imbalance is created 398 in the cells' nuclei. Importantly, this "reassignment" 399 process is underpinned by constraints on the extracellular 400 diffusion of $F_{N}$ molecules, and the constraints are 401 embodied only at the multicellular-individual level.

350 Finally, I assessed whether the total information 351 capacity (represented by $C\left(X_{1}, \ldots, X_{n}\right)$ in Eq. 1 and 352 measured in bits) of overall histone H3 crosstalk 353 (triad-wise) is significantly correlated with age, in ${ }_{354}$ particular, whether it is positively correlated. This 355 potential correlation is important, because if total 356 information capacity increases with cell donor age, 357 an age-correlated decrease of the proportion available 358 for epigenetic content would not be necessarily a 359 problem. That is, a proportionally smaller and smaller 360 information capacity for epigenetic content within histone 361 crosstalk would not generate an information content 362 imbalance - hypothesized in the Introduction - as long 363 as a growing total capacity provides enough room for 364 epigenetic content in absolute terms.

365 To test this possibility, the correlation value $r$ between 366 cell donor age and total information capacity (in bits) of 367 TSS-adjacent histone H3 crosstalk, computed as

$$
C_{Y}\left(X_{i}, X_{j}, X_{k}, Y\right)+C\left(X_{i}, X_{j}, X_{k} \mid Y\right)
$$

368 was obtained for all 551,300 triads of position-specific 369 histone $\mathrm{H} 3$ modifications for normal cells.

402 That is, in histone crosstalk there is a time-correlated ${ }_{403}$ loss of capacity for epigenetic information (i.e., less and 404 less epigenetic constraints on histone crosstalk), which ${ }_{405}$ causes a global and progressive impairment of biological 406 functions at the multicellular-individual level, eventually 407 causing the death of the individual.

${ }_{408}$ The nature of the epigenetic constraints on histone ${ }_{409}$ crosstalk strongly implicates this time-correlated loss 410 of capacity for epigenetic information content (and ${ }_{411}$ concurrent gain of that for hologenic content) as the 412 fundamental cause of senescence. Epigenetic constraints ${ }_{413}$ are explicitly related to transcriptional/gene expression ${ }_{414}$ changes and represented by the $C_{Y}\left(X_{1}, \ldots, X_{n}, Y\right)$ ${ }_{415}$ summand in Eq. 1. Because they depend on the ${ }_{416}$ interactions between the histone-modified nucleosomes ${ }_{417}$ and the DNA wrapped around them-allowing or 418 preventing transcription - the epigenetic information ${ }_{419}$ content they embody allows precise mRNA (and, 420 ultimately, gene expression) levels from histone ${ }_{421}$ modification patterns.

${ }_{422}$ This age-correlated hologenic/epigenetic information ${ }_{423}$ imbalance in histone crosstalk can also be understood The analysis revealed that the correlation coefficients $r \quad{ }^{424}$ in terms of an imbalance between the accuracy and
371 have mean, median, and standard deviation values $\bar{r}=0.21,{ }^{425}$ precision of transcription in the cells with respect to 371 have mean, median, and standard deviation values $\bar{r}=0.21,{ }^{425}$ the needs of the multicellular individual. That is, more
$372 \tilde{r}=0.21$, and $\sigma_{r}=0.24$, respectively, and that all associated ${ }^{426}$ the $372 \tilde{r}=0.21$, and $\sigma_{r}=0.24$, respectively, and that all associated ${ }^{426}$ the needs
${ }_{373} q$-values were equal to 1 and thus non-significant. ${ }^{427}$ accuracy (i.e., closeness of the mean mRNA level to the 374 For cancer cells, all correlation values were also ${ }^{428}$ mean level functional for the multicellular individual) is 375 non-significant $(q=1)$. Their mean, median, and standard ${ }^{429}$ reached with age at the expense of precision (i.e., closeness 376 deviation values were $\bar{r}=0.01, \tilde{r}=0.04$, and $\sigma_{r}=0.18,430$ of the resulting mRNA levels from the same pattern 
431 of histone modifications). This trade-off is unavoidable ${ }_{489}$ in the wild with the hazards it imposes [35]. However, 432 because (i) the relative growth of $C\left(X_{1}, \ldots, X_{n} \mid Y\right) 490$ under the falsifiable theory presented in this paper, this 433 implies an increasing constraint on (i.e., regulation 491 consensus is fundamentally incorrect. Indeed, senescence 434 of) histone modification patterns with respect to the 492 at the multicellular-individual level is, I suggest, not 435 multicellular individual [17], thus making transcription ${ }_{493}$ the result of relaxed selection but instead an intrinsic 436 more accurate and (ii) the concurrent relative decrease of ${ }_{494}$ developmental byproduct that would have been already ${ }_{437} C_{Y}\left(X_{1}, \ldots, X_{n}, Y\right)$ means histone modification patterns ${ }_{495}$ observable theoretically in the emergence of the very 438 become worse and worse predictors of mRNA levels, in ${ }_{496}$ first individuated multicellular organisms as described 439 turn making transcription less and less precise to the 497 by the hologenic theory [17]. In other words, had the ${ }_{440}$ point of dysfunctionality with respect to the multicellular ${ }_{498}$ first individuated multicellular organisms been free from ${ }_{441}$ individual (see schematic in Fig. 6a).

499 any extrinsic hazard in the wild, they would have begun

${ }_{442}$ Thus, we can characterize senescence under this theory 500 to senesce significantly after reaching a mature form in ${ }_{443}$ as a global transcriptional over-regulation with respect to ${ }_{501}$ their development, as opposed to displaying extremely 444 the multicellular individual's needs - as opposed to cancer, 502 slow or negligible senescence as can be inferred from the 445 where the dysfunctional effect is typically characterized ${ }_{503}$ relaxed-selection hypothesis.

446 in terms of the dysregulation of transcription and gene 504 If correct, the evolutionary account of senescence 447 expression $[28,29]$.

505 suggested here underscores the need for modern ${ }_{448}$ The following general prediction applies to the 506 evolutionary theory to incorporate the effects of the 449 falsifiability of the theory of senescence: Within genomic ${ }_{507}$ few yet crucial events where unprecedented forms of 450 regions adjacent to TSSs in primary normal cells from 508 biological individuality have emerged throughout the 451 any given tissue in any individuated multicellular species, 509 history of life on Earth. One of these events - as discussed 452 a significant positive correlation will be observed between 510 here - is the emergence of the individuated multicellular 453 the log-ratio of non-epigenetic to epigenetic histone 511 organism [17] with senescence as its developmental 454 crosstalk magnitude and the age of the individual 512 byproduct, and its influence on the population renewal 455 from whom the cells were obtained. The specific level ${ }_{513}$ process. Other emergence events where new forms of 456 of crosstalk - i.e., number of position-specific histone 514 individuality can arise with significant evolutionary 457 modifications involved - at which this correlation exists 515 consequences include the origin of life explicitly excluded ${ }_{458}$ may vary among species. It is predicted to be the level that 516 by Darwin from the scope of his original theory [36] — with ${ }_{459}$ possesses both significant predictive power and predictive ${ }_{517}$ its unprecedented self-regulating and self-reproducing 460 synergy (see Methods) on mRNA levels. Moreover, 518 dynamics that first enabled natural selection [44], and 461 since hologenic information content is described as 519 the emergence of the mind [37], which - through synthetic 462 emerging locally and independently in each developmental ${ }_{520}$ biology - could at some point elicit the appearance of ${ }_{463}$ process [17], the statistical strength of the predicted ${ }_{521}$ new species in the evolutionary process without any ${ }_{464}$ positive correlation will be further increased - and ${ }_{522}$ involvement of natural selection. These latter two events, 465 underpinned by a monotonically increasing function - if ${ }_{523}$ and potentially others, remain to be fully elucidated along 466 all primary cell samples are obtained from the same tissue 524 with their evolutionary consequences.

${ }_{467}$ of the same individual throughout its lifespan.

${ }_{525}$ Any theory of senescence is bound to address the ${ }_{468}$ The notable exceptions to be made for the prediction ${ }_{526}$ question of whether aging at the multicellular-individual 469 above are a few species able to undergo reverse ${ }_{527}$ level can be dynamically stopped. The answer suggested 470 developmental processes from adult to juvenile stages. 528 here is that achieving a dynamical arrest of senescence ${ }_{471}$ One such species is the jellyfish Turritopsis nutricula [30], 529 is not a fundamental impossibility but it may well 472 which is predicted to display an analogous negative 530 be a technical impossibility because of a therapeutic ${ }_{473}$ correlation in the processes, i.e., "reassignment" in ${ }_{531}$ safety issue. From a fundamental point of view, methods ${ }_{474}$ reverse. Another exception for the prediction are species 532 could be developed to, for example, artificially increase 475 displaying extremely slow or potentially negligible ${ }_{533}$ the dynamical range of nucleosome-DNA interactions 476 senescence processes [31]. Examples of these are the ${ }_{534}$ (thus increasing the capacity for epigenetic information 477 bristlecone pine Pinus longaeva [32], the freshwater ${ }_{535}$ content in histone crosstalk at the expense of that for ${ }_{478}$ polyp Hydra vulgaris [33], and the naked mole-rat 536 hologenic content).

479 Heterocephalus glaber [34], which, after adulthood, are ${ }_{537}$ Yet, the hologenic theory also predicts that a ${ }_{480}$ predicted to display a significant but very weak positive ${ }_{538}$ significant loss of hologenic content is a necessary 481 correlation (in cases where senescence is extremely slow), ${ }_{539}$ condition for the onset of cancer. If this is correct, 482 or an hologenic/epigenetic log-ratio invariant with age 540 a potentially unsurmountable safety problem arises: 483 (i.e., no correlation in cases where senescence is truly ${ }_{541}$ also under hologenic theory, the in vivo balance 484 negligible; Fig. 6b).

542 between hologenic and epigenetic information content ${ }_{485}$ Senescence is widely regarded as an evolutionary ${ }_{543}$ is predictably "fine-tuned" as it is individual-specific, ${ }_{486}$ consequence of the relaxation of selection on traits that ${ }_{544}$ cell-type-specific, and also confined to small functional ${ }_{487}$ maintain/repair the multicellular individual's functions in ${ }_{545}$ ranges. Thus, there could be an inherent high risk of 488 later life, because later life would have been rarely realized ${ }_{546}$ greatly increasing cancer incidence with the slightest 
bioRxiv preprint doi: https://doi.org/10.1101/310300; this version posted April 28, 2018. The copyright holder for this preprint (which was not certified by peer review) is the author/funder, who has granted bioRxiv a license to display the preprint in perpetuity. It is made available under 8 aCC-BY 4.0 International license.

a

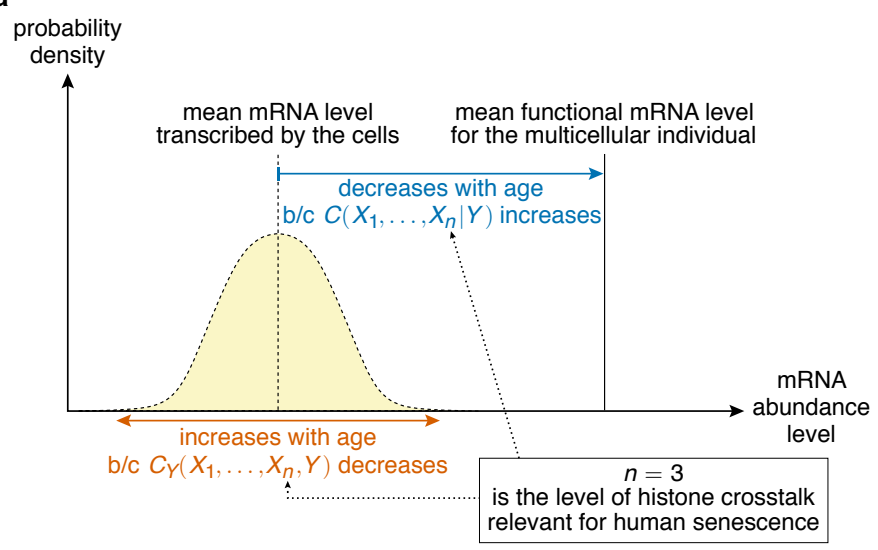

$C_{Y}\left(X_{1}, \ldots, X_{n}, Y\right)$

$C\left(X_{1}, \ldots, X_{n} \mid Y\right)$

$\rightarrow$ Conveys epigenetic information content $\bullet$ Conveys hologenic information content

-Allows precise mRNA levels with

-Decreases with age* $\quad$-Increases with age*

*see exceptions (blue and magenta $f(t))$ in panel b

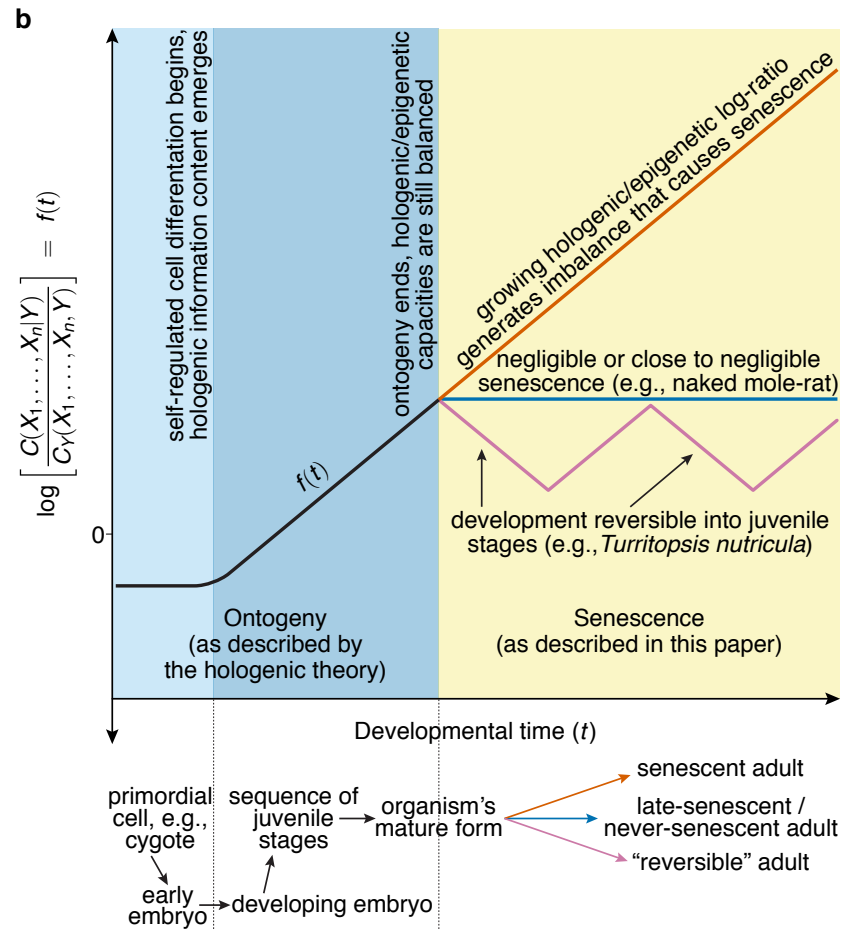

b

embryo

Fig. 6. Schematic of the imbalance between information capacity for hologenic content and epigenetic content in TSS-adjacent histone modification crosstalk as the cause of senescence. (a) The age-correlated increase in $C\left(X_{1}, \ldots, X_{n} \mid Y\right)$ and concurrent decrease in $C_{Y}\left(X_{1}, \ldots, X_{n}, Y\right)$ over-regulates the transcriptional response with respect to the multicellular individual's needs, i.e., the response becomes more and more accurate (blue) but less and less precise (orange) to the point of dysfunctionality. This unavoidable trade-off is explained by histone modification patterns becoming more constrained by regulation at the multicellular-individual level while at the same time becoming worse predictors of mRNA levels. The specific critical level of histone modification crosstalk (i.e., the value of $n$ in $\left\{X_{1}, \ldots, X_{n}\right\}$ ) at which this phenomenon occurs is $n=3$ (i.e., triads of position-specific histone modifications) in humans, but may vary for other species. (b) The log-ratio of non-epigenetic to epigenetic histone crosstalk magnitude increases during development as the embryo grows (black $f(t)$ in darker blue area). After the organism reaches its mature form (yellow area), the log-ratio continues to increase (orange $f(t)$ ) - with a few notable exceptions (blue and magenta $f(t)$ ). This continuous increase in turn creates an increasing dysfunctional imbalance of information contents that translates into senescence and, eventually, into death.

${ }_{547}$ extrinsic attempt to correct for the hologenic/epigenetic 571 individual against its own senescence. Although the 548 content imbalance. This problem resides in that hologenic 572 specific dynamics that would underpin the "pushback" 549 constraints, whose growth in magnitude has senescence 573 are beyond the scope of this paper, this hypothesis is 550 as a byproduct, are the very constraints preventing an 574 indeed falsifiable by means of the following secondary 551 otherwise likely onset of cancer [17].

575 prediction: the observed log-ratio of non-epigenetic to ${ }_{552}$ Based on a mathematical model of intercellular 576 epigenetic histone crosstalk magnitude in the normal 553 competition, Nelson and Masel have argued that stopping 577 (i.e., non-cancerous) cells closest to an age-related stage I 554 senescence, even if possible, will always elicit the onset of 578 malignant tumor will be significantly lower than said 555 cancer and that senescence is ultimately inevitable [38]. 579 log-ratio observed in the other (i.e., tumor-nonadjacent) 556 Nevertheless, the existence of individuated multicellular 580 normal cells of the same tissue. (Note: The falsification of ${ }_{557}$ species such as Turritopsis nutricula demonstrates that ${ }_{581}$ this secondary prediction does not imply the falsification 558 development can be reversed at least into juvenile ${ }_{582}$ of the theory as a whole.)

559 developmental stages [30] and that of the naked ${ }_{583}$ In turn, the "pushback"-against-senescence hypothesis 560 mole-rat suggests that senescence is reversible in some 584 for age-related cancer has, if correct, an implication we ${ }_{561}$ cases and negligible or close to negligible in others, 585 should not overlook. Namely, stopping senescence and 562 however exceptional.

586 eliminating the incidence of age-related cancer should be ${ }_{563}$ The delicate balance between hologenic and epigenetic ${ }_{587}$ one and the same technical challenge. In this respect, 564 information described here may shed light on the ${ }_{588}$ it is worth noting that in the naked mole-rat both 565 well-known positive correlation between cancer incidence 589 senescence [34] and cancer incidence [40,41] have been 566 and age [39]: if the senescent multicellular individual 590 described as negligible or close to negligible.

567 attempts to correct its growing hologenic/epigenetic 591 Rozhok and DeGregori have recently highlighted 568 content imbalance too strongly, it may elicit the onset 592 the explanatory limitations [42] of the Armitage-Doll 569 of cancer. Thus, age-related cancer would be the result ${ }_{593}$ multistage model of carcinogenesis, which regards the 570 of a strong enough "pushback" from the multicellular 594 accumulation of genetic mutations as the cause of 
595 age-related cancer [43]. They further argued that ${ }_{643}$ bigWigToWig $\rightarrow$ wig2bed --zero-indexed $\rightarrow$ 596 age-related cancer should rather be understood as a ${ }_{644}$ sort $-\mathrm{k} 1,1-\mathrm{k} 2,2 \mathrm{n} \rightarrow$ bedtools map $-\mathrm{o}$ median 597 function of senescence-related processes [42]. However, 598 their description of age-related cancer is based on ${ }^{645}$ ${ }_{599}$ Darwinian processes and thus differ from the account 600 suggested here, which can be understood within the 601 concept of teleodynamics $[37,44]$ - a framework of 602 biological individuality based on the emergence of intrinsic 603 higher-order constraints, such as that described in the 604 hologenic theory [17].

${ }_{605}$ Apart from the proof of concept presented here, if the 606 main prediction of this paper resists falsification attempts ${ }_{607}$ consistently, further research will be needed to elucidate 608 the specific molecular dynamics embodying hologenic and 609 epigenetic constraints within histone crosstalk completely. ${ }_{610}$ Such insights will be necessary to decide whether the ${ }_{611}$ hologenic/epigenetic information content imbalance can ${ }_{612}$ be corrected without compromising the multicellular ${ }_{613}$ individual's health or survival.

\section{METHODS}

\section{Data collection}

${ }_{614}$ The genomic coordinates and associated transcript 615 lengths of all annotated RefSeq mRNA TSSs for the hg19 ${ }_{616}$ (Homo sapiens) assembly were downloaded from the ${ }_{617}$ UCSC (University of California, Santa Cruz) database [45]. ${ }_{618}$ All ChIP-seq and RNA-seq data downloaded, processed, 619 and analyzed in this work were generated by the ${ }_{620}$ Canadian Epigenetics, Epigenomics, Environment and ${ }_{621}$ Health Research Consortium (CEEHRC) initiative ${ }_{622}$ funded by the Canadian Institutes of Health ${ }_{623}$ Research (CIHR), Genome BC, and Genome Quebec. ${ }_{624}$ CEEHRC protocols and standards can be found at 625 http://www.epigenomes.ca/protocols-and-standards, 626 and specific details on ChIP-seq antibody validation ${ }_{627}$ can be found on this link. Further information ${ }_{628}$ about the CEEHRC and the participating ${ }_{629}$ investigators and institutions can be found at ${ }_{630}$ http://www.cihr-irsc.gc.ca/e/43734.html. For a ${ }_{631}$ full list of source data files with their respective URLs ${ }_{632}$ for downloading, see Supplementary Information.

${ }_{633}$ Cell sample data sets in the CEEHRC database were 634 selected based on the following criteria: (i) only data ${ }_{635}$ sets with associated age were included and (ii) among 636 these data sets, the group (for both normal and cancer ${ }_{637}$ cells) that maximized the number of specific histone $\mathrm{H} 3$ ${ }_{638}$ modifications present in all data sets was chosen.

\section{ChIP-seq datafile processing}

-null 0 -a hg19_all_tss.bed/hg19_all_tss_control.bed ${ }_{646}$ to generate an associated BED (Browser Extensible Data) ${ }^{647}$ file. (Note: The hg19_all_tss. bed file is a 200bp-per-bin ${ }_{648}$ BED reference file with no score values to perform 649 the final ChIP-seq histone modification data mapping 650 onto the $6,000 \mathrm{bp}-$ long TSS-adjacent genomic regions. ${ }_{651}$ The hg19_all_control.bed file is an analogous BED ${ }_{652}$ reference file for mapping the ChIP-seq input data onto ${ }_{653}$ 200-bp, 1-kbp, 5-kbp, and 10-kbp genomic windows, 654 see ChIP-seq read profiles and normalization.)

\section{ChIP-seq read profiles and normalization}

To quantify and represent ChIP-seq read signal ${ }_{656}$ profiles for the histone H3 modifications, data were 657 processed with the same method used in the EFilter ${ }_{658}$ multivariate algorithm [23] to predict mRNA levels with 659 high accuracy $(R \sim 0.9)$. Steps in this method comprise ${ }_{660}$ (i) dividing the genomic region from $2 \mathrm{kbp}$ upstream ${ }_{661}$ to $4 \mathrm{kbp}$ downstream of each TSS into 30 200-bp-long 662 bins, in each of which ChIP-seq reads were later counted; ${ }_{663}$ (ii) dividing the read count signal for each bin by its 664 corresponding control (ChIP-seq input) read density to ${ }_{665}$ minimize artifactual peaks; (iii) estimating the control 666 read density within a 1-kbp window centered on each bin, ${ }_{667}$ if the 1-kbp window contained at least 20 reads; otherwise, ${ }_{668}$ a 5 -kbp window, or else a $10-\mathrm{kbp}$ window was used if the 669 control reads were less than 20 . When the 10 -kbp length 670 was insufficient, a pseudo-count value of 20 reads per 10 ${ }^{671} \mathrm{kbp}$ was set as the control read density. This implies that ${ }^{672}$ the denominator (i.e., control read density) is at least 0.4 673 reads per bin.

\section{RNA-seq datafile processing}

${ }_{674}$ For each strand in the DNA, original datafiles contained ${ }_{675}$ mRNA abundances in RPKM (reads per kilobase of 676 transcript per million mapped reads) in bigWig format. ${ }_{677}$ These datafiles were thus processed analogously to the ChIP-seq datafiles, i.e., using the pipeline

${ }_{679}^{678}$ bigWigToWig $\rightarrow$ wig2bed --zero-indexed $\rightarrow$

${ }_{680}$ sort $-k 1,1-k 2,2 n \rightarrow$ bedtools map -o median

-null 0 -a refseq_pos.bed/refseq_neg.bed

682 to obtain associated BED files. (Note: The ${ }_{683}$ refseq_pos.bed and refseq_neg.bed files are BED ${ }_{684}$ reference files for each strand, with no score values, to ${ }_{685}$ perform the final RPKM calculation for each RefSeq 686 mRNA in the hg19 assembly.)

${ }_{639}$ The original ChIP-seq binary datafile format was ${ }^{687}$ When two or more mRNAs shared the same TSS ${ }_{640}$ bigWig. For mapping its ChIP-seq signal into the hg19 ${ }_{688}$ (i.e., transcription start site with same genomic position ${ }_{641}$ assembly, each datafile was processed with standard ${ }^{689}$ and strand) the mean of the respective RPKM values was 642 bioinformatics tools [46-48] in the following pipeline:

690 computed and associated with the corresponding TSS. 
bioRxiv preprint doi: https://doi.org/10.1101/310300; this version posted April 28, 2018. The copyright holder for this preprint (which was not certified by peer review) is the author/funder, who has granted bioRxiv a license to display the preprint in perpetuity. It is made available under

\section{ChIP-seq/RNA-seq signal data tables}

${ }_{691}$ Using the RPKM values processed in this work, a 692 subset TSS $_{\text {def }}$ of all RefSeq mRNA TSSs displaying 72 ${ }_{693}$ measured abundance (i.e., RPKM $>0$ ) in all normal 722 statistical association were used in this work in order to 694 and cancer samples was determined. The number of 723 quantify histone H3 crosstalk at TSSs and its relationship ${ }_{695}$ TSSs in this subset TSS $_{\text {def }}$ was 18,220 , indicating that 724 with mRNA levels.

$696 \sim 70 \%$ of the 26,048 RefSeq mRNA TSSs annotated in 697 the hg19 assembly had an associated mRNA abundance 698 greater than zero in all (i.e., both normal and cancer) 699 samples. The obtained TSS def subset thus provided the 700 data analysis with a common basis for all samples that 701 comprises most protein-coding genes annotated in the 702 human genome.

${ }_{703}$ For each sample data entry, 30 genomic bins were 704 defined and denoted by the distance (bp) between their $7055^{\prime}$-end and their respective $\mathrm{TSS}_{\text {def }}$ genomic coordinate: 706 "-2000", "-1800", "-1600", "-1400", "-1200", "-1000", 707 "-800", "-600", "-400", "-200", "0" (TSS def or '+1'), 708 "200", "400", "600", "800", "1000", "1200", "1400", 709 "1600", "1800", "2000", "2200", "2400", "2600", "2800", 710 "3000", "3200", "3400", "3600", and "3800". Then, for 711 each sample data entry, the ChIP-seq read signal was 712 computed for all bins and for all histone modifications ${ }_{713}(30$ bins $\times 5$ modifications $=150$ signal values $)$ in all $\mathrm{TSS}_{\mathrm{def}}$ 714 genomic regions. Data input tables - comprising the 715 histone H3 modifications H3K4me1, H3K9me3, H3K27ac, ${ }_{716}$ H3K27me3, and H3K36me3 - were thus generated for 717 each sample entry as exemplified next:

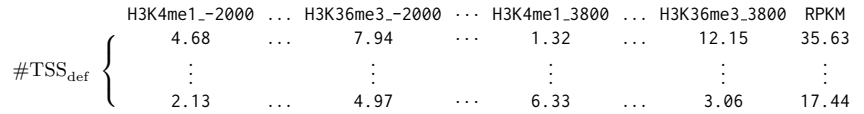

718 The tables were then written to tab-delimited datafiles, 719 which were subsequently classified into two groups: normal 720 and cancer cells (see Table $\mathbf{1}$ ).

\begin{tabular}{|c|c|}
\hline group & datafiles \\
\hline $\begin{array}{c}\text { normal } \\
(n=18)\end{array}$ & $\begin{array}{l}\text { CEMT0032.nm.dat CEMT0033.nm.dat } \\
\text { CEMT0034.nm.dat CEMT0040.nm.dat } \\
\text { CEMT0042.nm.dat CEMT0044.nm.dat } \\
\text { CEMT0050.nm.dat CEMT0051.nm.dat } \\
\text { CEMT0052.nm.dat CEMT0053.nm.dat } \\
\text { CEMT0054.nm.dat CEMT0055.nm.dat } \\
\text { CEMT0056.nm.dat CEMT0057.nm.dat } \\
\text { CEMT0058.nm.dat CEMT0059.nm.dat } \\
\text { CEMT0060.nm.dat CEMT0061.nm.dat }\end{array}$ \\
\hline $\begin{array}{c}\text { cancer } \\
(n=17)\end{array}$ & $\begin{array}{l}\text { CEMT0004.nm.dat CEMT0005.nm.dat } \\
\text { CEMT0006.nm.dat CEMT0019.nm.dat } \\
\text { CEMT0021.nm.dat CEMT0025.nm.dat } \\
\text { CEMT0026.nm.dat CEMT0027.nm.dat } \\
\text { CEMT0028.nm.dat CEMT0029.nm.dat } \\
\text { CEMT0030.nm.dat CEMT0047.nm.dat } \\
\text { CEMT0063.nm.dat CEMT0064.nm.dat } \\
\text { CEMT0065.nm.dat CEMT0066.nm.dat } \\
\text { CEMT0067.nm.dat }\end{array}$ \\
\hline
\end{tabular}

Table 1. Datafiles generated in this work containing normalized ChIP-seq signal values and RPKM values. The ' $\mathrm{nm}$ ' suffix in the filename refers to the 'NM' RefSeq label for messenger RNAs, as opposed to non-coding RNAs.

\section{Shannon measures of statistical uncertainty and statistical association}

\section{Statistical uncertainty}

725 C.E. Shannon's seminal work, among other things, 726 introduced the notion of - and a measure for - the 727 uncertainty about discrete random variables [49]. For a ${ }_{728}$ discrete random variable $\mathrm{X}$ with probability mass function ${ }_{729} P(X)$ its uncertainty (also known as Shannon entropy) is 730 defined as

$$
H(X):=-\sum_{x \in X} P(x) \log _{b}[P(x)]
$$

731 where $P(x)$ is the probability of $X=x$ and $b$ is the 732 logarithm base. When $b=2$ (the base used in this work), 733 the unit for this measure is the bit. $H(X)$ can also 734 be interpreted as the amount of information necessary 735 to resolve the uncertainty about the outcome of $X$. ${ }_{736}$ Shannon uncertainty was the measure used to estimate 737 the uncertainty about the mRNA abundance level to be ${ }_{738}$ resolved in normal cells.

$739 H(X)$ is typically called marginal uncertainty because 740 it involves only one random variable. In a multivariate 741 scenario, the measure $H\left(X_{1}, \ldots, X_{n}\right)$ is called the joint 742 uncertainty of the set of discrete random variables $743\left\{X_{1}, \ldots, X_{n}\right\}$, and it is analogously defined as

$$
H\left(X_{1}, \ldots, X_{n}\right):=-\sum_{x \in X_{1}} \cdots \sum_{x \in X_{n}} P\left(x_{1}, \ldots, x_{n}\right) \log _{b}\left[P\left(x_{1}, \ldots, x_{n}\right)\right] .
$$
745 uncertainty about a discrete random variable $Y$, with 746 probability mass function $P(Y)$, given that the value 747 of another discrete random variable $X$ is known. ${ }_{748}$ This conditional uncertainty $H(Y \mid X)$ can be expressed 749 as

$$
H(Y \mid X)=-\sum_{x \in X} \sum_{y \in Y} P(x, y) \log _{b}\left[\frac{P(x)}{P(x, y)}\right]
$$

750 where $P(x, y)$ is the joint probability of $X=x$ and $Y=y$. 751 Importantly, any measure of Shannon uncertainty (or any 752 other derived Shannon measure) that is conditional on a ${ }_{753}$ random variable $X$ can also be understood as said measure 754 being explicitly unrelated to, or statistically independent 755 from, the variable $X$.
744 Another measure important to this work is the conditional 


\section{Statistical association}

756 A classic Shannon measure of statistical association of 757 any two discrete random variables $X$ and $Y$ is that of 758 mutual information $I$, defined as

$$
\begin{aligned}
I(X ; Y) & :=-\sum_{x \in X} \sum_{y \in Y} P(x, y) \log _{b}\left[\frac{P(x, y)}{P(x) P(y)}\right] \\
& =H(X)+H(Y)-H(X, Y) \\
& =H(Y)-H(Y \mid X) .
\end{aligned}
$$

759 Note that if and only if $X$ and $Y$ are statistically 760 independent then $I(X ; Y)=0, H(X, Y)=H(X)+H(Y)$, 761 and $H(Y \mid X)=H(Y)$. To analyze the magnitude of 762 histone H3 crosstalk at TSSs, the two best known 763 multivariate generalizations of mutual information were 764 used in this work. The first is interaction information [50] 765 or co-information [51], also symbolized by $I$, which is 766 defined analogously to Eq. 8 for a set $V$ of $n$ discrete 767 random variables as

$$
I(V):=\sum_{U \subseteq V}(-1)^{|U|+1} H(U),
$$

768 where $|U|$ is the cardinality (in this case, the number 769 of random variables) of the subset $U$. In the case of 770 interaction information $I$, Shannon uncertainty $H$ is thus 771 summed over all subsets of $V$ (the uncertainty of the 772 empty subset is $H(\varnothing)=0)$. Importantly, the interaction 773 information of the random variables $\left\{X_{1}, \ldots, X_{n}\right\}$ can be 774 decomposed with respect to another random variable $Y$ 775 as follows:

$$
I\left(X_{1} ; \ldots ; X_{n}\right)=I\left(X_{1} ; \ldots ; X_{n} ; Y\right)+I\left(X_{1} ; \ldots ; X_{n} \mid Y\right) .
$$

776 Interaction information $I\left(X_{1} ; \ldots ; X_{n}\right)$ captures the 777 statistical association of all variables $\left\{X_{1}, \ldots, X_{n}\right\}$ taken 778 at once, i.e., excluding all lower-order associations, and it 779 can also take negative values in some cases. Interaction 780 information was used in this work as a means to compute 781 total correlation values.

${ }_{782}$ To specifically quantify the magnitude of histone H3 783 crosstalk, the second multivariate generalization of mutual 784 information used in this work was total correlation [24] 785 (symbolized by $C$ ) or multiinformation [25], which is 786 defined as

$$
C\left(X_{1}, \ldots, X_{n}\right):=\left[\sum_{i=1}^{n} H\left(X_{i}\right)\right]-H\left(X_{1}, \ldots, X_{n}\right),
$$

787 i.e., as the sum of the marginal uncertainties of the random 788 variables $\left\{X_{1}, \ldots, X_{n}\right\}$ minus their joint uncertainty 789 Importantly, and unlike interaction information $I$, total 790 correlation $C$ captures all possible statistical associations 791 including lower-order associations or, equivalently, all 792 possible associations between any two or more random 793 variables in the set $\left\{X_{1}, \ldots, X_{n}\right\}$. This is because the 794 definition of interaction information $I$ in Eq. 10 allows
795 total correlation $C$ to be rewritten as a sum of quantities $I$ 796 for all possible combinations of variables in $\left\{X_{1}, \ldots, X_{n}\right\}$ :

$$
C\left(X_{1}, \ldots, X_{n}\right)=\sum_{i, j} I\left(X_{i} ; X_{j}\right)+\sum_{i, j, k} I\left(X_{i} ; X_{j} ; X_{k}\right)+\ldots+I\left(X_{1} ; \ldots ; X_{n}\right) .
$$

${ }_{797}$ This expression for total correlation $C$ as a sum of 798 interaction information quantities $I$ along with the sum 799 decomposition of $I$ in Eq. 11 allows $C$ to be decomposed 800 also as a sum:

$$
C\left(X_{1}, \ldots, X_{n}\right)=C_{Y}\left(X_{1}, \ldots, X_{n}, Y\right)+C\left(X_{1}, \ldots, X_{n} \mid Y\right),
$$

801 where $C_{Y}\left(X_{1}, \ldots, X_{n}, Y\right)$ is the sum (analogous to that ${ }_{802}$ of Eq. 13) of all interaction information quantities $I$ but 803 now including the random variable $Y$ in each combination 804 of variables in $\left\{X_{1}, \ldots, X_{n}\right\}$, i.e.,

$$
C_{Y}\left(X_{1}, \ldots, X_{n}, Y\right)=\sum_{i, j} I\left(X_{i} ; X_{j} ; Y\right)+\sum_{i, j, k} I\left(X_{i} ; X_{j} ; X_{k} ; Y\right)+\ldots+I\left(X_{1} ; \ldots ; X_{n} ; Y\right),
$$

805 and where $C\left(X_{1}, \ldots, X_{n} \mid Y\right)$ is the sum of all conditional 806 interaction information quantities $I$ given $Y$ for each 807 combination of variables in $\left\{X_{1}, \ldots, X_{n}\right\}$, i.e.,

$$
C\left(X_{1}, \ldots, X_{n} \mid Y\right)=\sum_{i, j} I\left(X_{i} ; X_{j} \mid Y\right)+\sum_{i, j, k} I\left(X_{i} ; X_{j} ; X_{k} \mid Y\right)+\ldots+I\left(X_{1} ; \ldots ; X_{n} \mid Y\right) .
$$

For this work's purposes, total correlation $C$ was 809 chosen as the measure of statistical association to 810 assess TSS-adjacent histone crosstalk because (i) $C$ is 811 non-negative and thus easier to interpret conceptually, 812 (ii) $C$ is equal to zero if and only if all random variables it ${ }_{813}$ comprises are statistically independent, (iii) $C$ captures 814 all possible associations up to a given number of variables 815 (in this work, position-specific histone modification levels) 816 and, (iv) $C$ can be decomposed, as shown in Eq. 14, 817 as a sum of two $C$ quantities: one explicitly related to 818 a certain variable $Y$ and the other explicitly unrelated 819 to $Y$. Property (iv) was useful to decompose the overall 820 histone crosstalk as a sum of an epigenetic and other 821 non-epigenetic component (see Introduction).

An additional Shannon measure of statistical 823 association was used to assess the predictive power ${ }_{824}$ of TSS-adjacent histone modification levels on mRNA 825 abundance levels (such power has already been used 826 to predict mRNA levels with high accuracy [23]). ${ }_{827}$ The uncertainty coefficient $U[52]$ is defined as

$$
U\left(Y \mid X_{1}, \ldots, X_{n}\right):=\frac{H(Y)-H\left(Y \mid X_{1}, \ldots, X_{n}\right)}{H(Y)},
$$

828 i.e., $U\left(Y \mid X_{1}, \ldots, X_{n}\right)$ is the relative decrease in 829 uncertainty about $Y$ when $\left\{X_{1}, \ldots, X_{n}\right\}$ are known-or, 830 equivalently, the fraction of bits in $Y$ that can be predicted 831 by $\left\{X_{1}, \ldots, X_{n}\right\}$-and it can take values from 0 to 1 . ${ }_{832} U\left(Y \mid X_{1}, \ldots, X_{n}\right)=0$ implies the set $\left\{X_{1}, \ldots, X_{n}\right\}$ has 833 no predictive power on $Y$, whereas $U\left(Y \mid X_{1}, \ldots, X_{n}\right)=1$ 834 implies $\left\{X_{1}, \ldots, X_{n}\right\}$ can predict $Y$ completely. 
Levels of possible statistical associations when assessing histone crosstalk magnitudes
868 substantially lower $\left(U\left(Y \mid X_{i}, X_{j}\right)=0.07\right)$. On the other 869 hand, tetrads (i.e., when $n=4$ ) were found to have high 870 predictive power $\left(U\left(Y \mid X_{i}, X_{j}, X_{k}, X_{l}\right)=0.95\right)$ but they 871 possess no synergy whatsoever and display instead what is 872 called redundancy [53]. Based on previous work [23], high 873 predictive power on mRNA levels and yet no synergy are 874 thus expected to happen with a large enough $n$. From all 875 possible singletons (4), pairs (6), and triads (4) that exist 876 within a tetrad, the explanatory power on mRNA levels ${ }_{841}$ To this end, the minimal $n$ able to predict mRNA 877 of a non-redundant set of only one triad and five pairs
${ }_{842}$ levels significantly and non-redundantly-which 878 already exceeds the explanatory power of the tetrad. ${ }_{841}$ To this end, the minimal $n$ able to predict mRNA 877 of a non-redundant set of only one triad and five pairs
${ }_{842}$ levels significantly and non-redundantly-which 878 already exceeds the explanatory power of the tetrad. ${ }_{843}$ corresponds to the level of histone crosstalk able 879 (Note: In previous work it has been argued that RPKM 844 to convey a non-neglectable amount of epigenetic 880 may not always be a suitable unit of mRNA abundance 845 information content-was first determined. This value is 881 when studying differential gene expression. Specifically, 846 straightforward to assess using the uncertainty coefficient 882 it was shown that, if transcript size distribution ${ }_{847} U\left(Y \mid X_{1}, \ldots, X_{n}\right)$, where $Y$ represents mRNA levels.

883 varies significantly among the samples, RPKM might ${ }_{848}^{84}$ In effect, $U\left(Y \mid X_{i}, X_{j}\right)$ (i.e., where $n=2$ ) quantifies 884 introduce significant biases [55]. To overcome this problem,
849 the predictive power of pairs of position-specific 885 an alternative abundance unit TPM (transcripts per 850 histone modification levels, $U\left(Y \mid X_{i}, X_{j}, X_{k}\right)$ quantifies 886 million)-which is an invertible linear transformation of ${ }_{851}$ the predictive power of triads, etc. $U\left(Y \mid X_{1}, \ldots, X_{n}\right){ }_{887}$ the RPKM value for each sample-was introduced [55]. ${ }_{852}$ values were thus computed for singletons, pairs, triads, 888 Nonetheless, this issue was not a problem for the 853 and tetrads. Singeltons were calculated for descriptive 889 present work because Shannon measures are invariant ${ }_{854}$ purposes only, because histone crosstalk is not measurable 890 under any invertible transformation of the discrete 855 for them. On average, a triad (i.e., when $n=3$ ) of 891 random variables.)

856 position-specific histone H3 modification levels was found 857 to have (i) significant predictive power on mRNA levels ${ }_{858}\left(U\left(Y \mid X_{i}, X_{j}, X_{k}\right)=0.63\right)$ and, importantly, (ii) at least 2.3 859 times more predictive power than all possible singletons ${ }_{860}(3)$ and pairs (3) that exist within a triad taken together, 861 i.e.,

$$
\frac{U\left(Y \mid X_{i}, X_{j}, X_{k}\right)}{\sum_{i} U\left(Y \mid X_{i}\right)+\sum_{i, j} U\left(Y \mid X_{i}, X_{j}\right)} \geq 2.3,
$$

${ }_{862}$ a phenomenon known as synergy of a set of 863 predictor variables [53] (see Table 2).

\begin{tabular}{|c|c|c|c|c|}
\hline level & set & $\boldsymbol{U}(\boldsymbol{Y} \mid \boldsymbol{V})[$ mean $]$ & $\boldsymbol{\kappa}$ & $\boldsymbol{U}(\boldsymbol{Y} \mid \boldsymbol{V})$ measured in \\
\hline singleton & $V=\left\{X_{i}\right\}$ & 0.02 & 154 & all 150 singletons \\
\hline pair & $V=\left\{X_{i}, X_{j}\right\}$ & 0.07 & 441 & all 11,175 pairs \\
\hline triad & $V=\left\{X_{i}, X_{j}, X_{k}\right\}$ & 0.63 & 325 & all 551,300 triads \\
\hline tetrad & $V=\left\{X_{i}, X_{j}, X_{k}, X_{l}\right\}$ & 0.95 & 379 & 50,000 random tetrads \\
\hline
\end{tabular}

Table 2. Predictive power (quantified as $U(Y \mid V) \in[0,1]$ ) of different sets $V=\left\{X_{1}, \ldots, X_{n}\right\}$ of TSS-adjacent, position-specific histone H3 modification levels on mRNA levels (represented by $\boldsymbol{Y}$ ) in normal cells. For each instance of the set $V, U(Y \mid V)$ was averaged over the 18 normal cell samples analyzed. The distribution of $U(Y \mid V)$ in the interval $[0,1]$ for each family $V$ of sets (singletons, pairs, triads, and tetrads) was parameterized and described here in terms of the mean $\bar{x}$ and the concentration parameter $\kappa$, which are derived from the respective beta distribution $\operatorname{Beta}(\alpha, \beta)$, with $\kappa=\hat{\alpha}+\hat{\beta}$ [54]. Point estimators $\hat{\alpha}$ and $\hat{\beta}$ were computed using the method of moments, i.e., $\hat{\alpha}=\bar{x}^{2}\left(\frac{1-\bar{x}}{\sigma^{2}}-\frac{1}{\bar{x}}\right)$ and $\hat{\beta}=\hat{\alpha}\left(\frac{1}{\bar{x}}-1\right)$, where $\sigma^{2}$ is the variance.

${ }_{864}$ Pairs (i.e., when $n=2$ ) were also found to possess 865 predictive synergy, but this synergy is smaller than that 866 found for triads $\left(1.75 \leq \frac{U\left(Y \mid X_{i}, X_{j}\right)}{\sum_{i} U\left(Y \mid X_{i}\right)}<2.3\right)$. The average ${ }_{867}$ predictive power of pairs on mRNA levels is also

\section{Theoretical methods}

The elaboration of the main falsifiable prediction took 893 into account two observations for human primary cells 894 in this work. Namely, (i) the uniqueness of triads of 895 position-specific histone modification levels in terms of 896 significant predictive power and predictive synergy and ${ }_{897}$ (ii) the post hoc result that triads constitute precisely the 898 level $n$ at which the predicted correlation between the 899 non-epigenetic/epigenetic histone H3 crosstalk log-ratio 900 and cell donor age actually exists. In this way, the main 901 prediction was formulated with explicit dependence on the 902 level of scale: "For any given tissue in any individuated 903 multicellular species a positive correlation between the 904 non-epigenetic/epigenetic histone H3 crosstalk log-ratio 905 and cell donor age will be observed at the level $n$ of histone 906 crosstalk that possesses both significant predictive power 907 and predictive synergy on mRNA levels."

\section{Statistical tests}

The statistical significance of each Pearson correlation 909 coefficient $r$ obtained was assessed using the statistic $t$ 910 defined as

$$
t:=r \sqrt{\frac{n-2}{1-r^{2}}},
$$

911 which is known to follow a Student's $t$-distribution 912 with $n-2$ degrees of freedom, and where $n$ is the 913 number of data pairs [56]. For the hypothesized ${ }_{914}$ positive correlation between the non-epigenetic/epigenetic 
915 histone H3 crosstalk log-ratio and age, the statistical null 916 hypothesis was tested against the alternative hypothesis 917 that the correlation is greater than zero (i.e., one-sided ${ }_{918}$ Student's $t$-test). For the hypothesized non-significant ${ }_{919}$ correlation between the overall histone H3 crosstalk 920 magnitude and age, the statistical null hypothesis ${ }_{921}$ was tested against the alternative hypothesis that the ${ }_{922}$ correlation is greater or less than zero (i.e., two-sided ${ }_{923}$ Student's $t$-test).

${ }_{924}$ On the other hand, the distribution of correlation 925 coefficients $(r)$ is known to be non-Gaussian [57], which 926 can be easily appreciated in Fig. 3. For this reason, the 927 statistical comparison of $r$ for normal cells (neonate data ${ }_{928}$ point excluded) and cancer cells was performed using the 929 non-parametric Mann-Whitney $U$ test [58].

\section{Correction for multiple testing}

930 The analysis of histone crosstalk involved 9315 histone $\mathrm{H} 3$ modifications $\times 30$ genomic bins $=150$ ${ }_{932}$ TSS-adjacent, position-specific histone H3 modification ${ }_{933}$ levels. Thus, assessing the statistical significance of the ${ }_{934}$ correlation values involved a large number of tests of ${ }_{935}$ the null hypothesis (for triads, tetrads, and pairs) under 936 general dependence. This dependence derives from the 937 fact that different histone modification levels are known ${ }_{938}$ to be highly correlated (this is the phenomenon of histone 939 crosstalk itself).

940 The resampling-based procedure by Benjamini and ${ }_{941}$ Yekutieli [59] provides control of the false discovery rate ${ }_{942}$ (FDR) [60] under general dependence conditions. This was ${ }_{943}$ the method thus used in this work in order to correct for 944 multiple testing.

\section{Code availability}

945 Standard bioinformatics tools [46-48] and the Perl 946 language were used to process the ChIP-seq and RNA-seq ${ }_{947}$ source data and to generate the *. nm. dat files displayed in ${ }_{948}$ Table 1 . The R software [61] and its infotheo package [62] 949 were used for the computation of Shannon measures of 950 statistical uncertainty and statistical association from the 951 *. nm. dat files. Marginal and joint Shannon uncertainties 952 and all the other derived Shannon measures were 953 computed using maximum likelihood (ML) estimation [63] 954 and bias-corrected with the Miller-Madow method [64]. 955 All the $\mathrm{R}$ code and the *.nm. dat files necessary for 956 a full reproduction of the results are available as 957 Supplementary Information.

\section{Sample metadata}

\begin{tabular}{|c|c|c|c|c|}
\hline sample ID & disease status & cell donor age & $\operatorname{sex}$ & cell type/tissue \\
\hline CEMT0032 & normal & 0 & $\mathrm{~N} / \mathrm{A}$ & hematopoietic (cord) \\
\hline CEMT0033 & normal & 82 & $\mathrm{~F}$ & colon \\
\hline CEMT0034 & normal & 73 & M & colon \\
\hline CEMT0040 & normal & 67 & $\mathrm{~F}$ & thyroid \\
\hline CEMT0042 & normal & 46 & $\mathrm{~F}$ & thyroid \\
\hline CEMT0044 & normal & 55 & $\mathrm{M}$ & thyroid \\
\hline CEMT0050 & normal & 53 & $\mathrm{M}$ & colon \\
\hline CEMT0051 & normal & 67 & M & colon \\
\hline CEMT0052 & normal & 72 & $\mathrm{M}$ & colon \\
\hline CEMT0053 & normal & 81 & $\mathrm{~F}$ & colon \\
\hline CEMT0054 & normal & $90+$ & $\mathrm{F}$ & colon \\
\hline CEMT0055 & normal & $90+$ & $\mathrm{F}$ & colon \\
\hline CEMT0056 & normal & 84 & $\mathrm{~F}$ & colon \\
\hline CEMT0057 & normal & 84 & $\mathrm{~F}$ & colon \\
\hline CEMT0058 & normal & 56 & $\mathrm{~F}$ & colon \\
\hline CEMT0059 & normal & 56 & $\mathrm{~F}$ & colon \\
\hline CEMT0060 & normal & 77 & M & colon \\
\hline CEMT0061 & normal & 77 & M & colon \\
\hline CEMT0004 & cancer & 74 & M & peripheral blood \\
\hline CEMT0005 & cancer & 68 & $\mathrm{~F}$ & peripheral blood \\
\hline CEMT0006 & cancer & 60 & M & peripheral blood \\
\hline CEMT0019 & cancer & 46 & $\mathrm{~F}$ & brain \\
\hline CEMT0021 & cancer & 35 & M & brain \\
\hline CEMT0025 & cancer & 62 & $\mathrm{~F}$ & peripheral blood \\
\hline CEMT0026 & cancer & 79 & $\mathrm{M}$ & peripheral blood \\
\hline CEMT0027 & cancer & 71 & $\mathrm{M}$ & peripheral blood \\
\hline CEMT0028 & cancer & 79 & $\mathrm{~F}$ & peripheral blood \\
\hline CEMT0029 & cancer & 47 & M & colon \\
\hline CEMT0030 & cancer & 56 & $\mathrm{~F}$ & peripheral blood \\
\hline CEMT0047 & cancer & 43 & M & brain \\
\hline CEMT0063 & cancer & 81 & $\mathrm{~F}$ & colon \\
\hline CEMT0064 & cancer & $90+$ & $\mathrm{F}$ & colon \\
\hline CEMT0065 & cancer & 84 & $\mathrm{~F}$ & colon \\
\hline CEMT0066 & cancer & 56 & $\mathrm{~F}$ & colon \\
\hline CEMT0067 & cancer & 77 & M & colon \\
\hline
\end{tabular}

Table 3. Metadata for each primary cell sample analyzed. Note: Age entries originally tabulated as $90+$ were entered as 90 into the computational analysis. Metadata source: CEEHRC.

\section{ACKNOWLEDGMENTS}

I wish to thank Angelika H. Hofmann (1) at SciWri Services for editing this paper into an English I could only hope to write. 


\section{REFERENCES}

[1] S. Katz, Imagining the life-span: from premodern miracles to postmodern fantasies, in: Images of Aging: Cultural Representations of Later Life, Routledge, 1995, pp. 59-74.

[2] J. Vijg, J. Campisi, Puzzles, promises and a cure for ageing, Nature 454 (2008) 1065-1071. DOI:10.1038/nature07216.

[3] J. W. Shay, W. E. Wright, Hayflick, his limit, and cellular ageing, Nat. Rev. Mol. Cell Biol. 1 (2000) 72-76. DOI: 10.1038/35036093.

[4] L. Hayflick, P. S. Moorhead, The serial cultivation of human diploid cell strains, Exp. Cell Res. 25 (3) (1961) 585-621. DOI : 10.1016/0014-4827(61)90192-6.

[5] C. B. Harley, A. B. Futcher, C. W. Greider, Telomeres shorten during ageing of human fibroblasts, Nature 345 (1990) 458-460. DOI: 10.1038/345458a0.

[6] R. C. Allsopp, H. Vaziri, C. Patterson, S. Goldstein, E. V. Younglai, A. B. Futcher, C. W. Greider, C. B. Harley, Telomere length predicts replicative capacity of human fibroblasts, Proc. Natl. Acad. Sci. U.S.A. 89 (21) (1992) 10114-10118. DOI:10.1073/pnas.89.21.10114.

[7] V. J. Cristofalo, R. G. Allen, R. J. Pignolo, B. G. Martin, J. C. Beck, Relationship between donor age and the replicative lifespan of human cells in culture: a reevaluation, Proc. Natl. Acad. Sci. U.S.A. 95 (18) (1998) 10614-10619. DOI : 10.1073/pnas.95.18.10614.

[8] A. Lorenzini, M. Tresini, S. N. Austad, V. J. Cristofalo, Cellular replicative capacity correlates primarily with species body mass not longevity, Mech. Ageing Dev. 126 (10) (2005) 1130-1133. DOI:10.1016/j.mad.2005.05. 004.

[9] C. López-Otín, M. A. Blasco, L. Partridge, M. Serrano, G. Kroemer, The hallmarks of aging, Cell 153 (6) (2013) 1194-1217. DOI:10.1016/j.cell.2013.05.039.

[10] K. Jin, Modern biological theories of aging, Aging Dis. 1 (2) (2010) 72-74. Available from: http://www. aginganddisease.org/EN/Y2010/V1/I2/72.

[11] M. V. Blagosklonny, Aging is not programmed: genetic pseudo-program is a shadow of developmental growth, Cell Cycle 12 (24) (2013) 3736-3742. DOI:10.4161/cc. 27188.

[12] S. Horvath, K. Raj, DNA methylation-based biomarkers and the epigenetic clock theory of ageing, Nat. Rev. Genet. (2018) DOI: 10.1038/s41576-018-0004-3.

[13] R. W. Powers 3rd, M. Kaeberlein, S. D. Caldwell, B. K. Kennedy, S. Fields, Extension of chronological life span in yeast by decreased TOR pathway signaling, Genes Dev. 20 (2) (2006) 174-184. DOI:10.1101/gad.1381406.

[14] T. Vellai, K. Takacs-Vellai, Y. Zhang, A. L. Kovacs, L. Orosz, F. Müller, Genetics: Influence of TOR kinase on lifespan in C. elegans, Nature 426 (2003) 620. DOI: 10.1038/426620a.

[15] K. Popper, The Logic of Scientific Discovery, Routledge, 2005.

[16] G. Ellis, J. Silk, Scientific method: defend the integrity of physics, Nat. News 516 (7531) (2014) 321-323. Available from: http://www. nature.com/news/ scientific-method-defend-the-integrity-of-physics-1. 16535.

[17] F. A. Veloso, On the developmental self-regulatory dynamics and evolution of individuated multicellular organisms, J. Theor. Biol. 417 (2017) 84-99. DOI:10.1016/ j.jtbi.2016.12.025.
[18] P. W. Atkins, The Second Law, Scientific American Library, 1984.

[19] J.-S. Lee, E. Smith, A. Shilatifard, The language of histone crosstalk, Cell 142 (5) (2010) 682-685. DOI:10.1016/j. cell.2010.08.011.

[20] Z. Wang, C. Zang, J. A. Rosenfeld, D. E. Schones, A. Barski, S. Cuddapah, K. Cui, T.-Y. Roh, W. Peng, M. Q. Zhang, K. Zhao, Combinatorial patterns of histone acetylations and methylations in the human genome, Nat. Genet. 40 (7) (2008) 897-903. DOI:10.1038/ng. 154.

[21] A. Lennartsson, K. Ekwall, Histone modification patterns and epigenetic codes, Biochim. Biophys. Acta - Gen. Subj. 1790 (9) (2009) 863-868. DOI:10.1016/j.bbagen.2008.12. 006.

[22] V. E. A. Russo, R. A. Martienssen, A. D. Riggs, Epigenetic Mechanisms of Gene Regulation, Cold Spring Harbor Laboratory Press, 1996.

[23] V. Kumar, M. Muratani, N. A. Rayan, P. Kraus, T. Lufkin, H. H. Ng, S. Prabhakar, Uniform, optimal signal processing of mapped deep-sequencing data, Nat. Biotechnol. 31 (7) (2013) 615-622. DOI:10.1038/nbt.2596.

[24] S. Watanabe, Information theoretical analysis of multivariate correlation, IBM J. Res. Dev. 4 (1) (1960) 66-82. DOI : 10.1147/rd.41.0066.

[25] M. Studený, J. Vejnarova, The multiinformation function as a tool for measuring stochastic dependence, in: Learning in Graphical Models, 1998, pp. 261-297. DOI:10.1007/ 978-94-011-5014-9_10.

[26] G. Csárdi, A. Franks, D. S. Choi, E. M. Airoldi, D. A. Drummond, Accounting for experimental noise reveals that mRNA levels, amplified by post-transcriptional processes, largely determine steady-state protein levels in yeast, PLoS Genet. 11 (5) (2015) e1005206. DOI:10.1371/journal.pgen. 1005206.

[27] S. E. Prochnik, J. Umen, A. M. Nedelcu, A. Hallmann, S. M. Miller, I. Nishii, P. Ferris, A. Kuo, T. Mitros, L. K. Fritz-Laylin, U. Hellsten, J. Chapman, O. Simakov, S. A. Rensing, A. Terry, J. Pangilinan, V. Kapitonov, J. Jurka, A. Salamov, H. Shapiro, J. Schmutz, J. Grimwood, E. Lindquist, S. Lucas, I. V. Grigoriev, R. Schmitt, D. Kirk, D. S. Rokhsar, Genomic analysis of organismal complexity in the multicellular green alga Volvox carteri, Science 329 (5988) (2010) 223-226. DOI:10.1126/science. 1188800.

[28] K. Malik, K. W. Brown, Epigenetic gene deregulation in cancer, Br. J. Cancer 83 (12) (2000) 1583-1588. DOI: 10.1054/bjoc. 2000.1549.

[29] T. J. Gonda, R. G. Ramsay, Directly targeting transcriptional dysregulation in cancer, Nat. Rev. Cancer 15 (11) (2015) 686-694. DOI:10.1038/nrc4018.

[30] S. Piraino, F. Boero, B. Aeschbach, V. Schmid, Reversing the life cycle: medusae transforming into polyps and cell transdifferentiation in Turritopsis nutricula (Cnidaria, Hydrozoa), Biol. Bull. 190 (3) (1996) 302-312. DOI : 10.2307/1543022.

[31] C. E. Finch, Update on slow aging and negligible senescence-a mini-review, Gerontology 55 (3) (2009) 307-313. DOI : 10.1159/000215589.

[32] R. M. Lanner, K. F. Connor, Does bristlecone pine senesce?, Exp. Gerontol. 36 (4-6) (2001) 675-685. DOI: 10.1016/S0531-5565(00)00234-5.

[33] R. Schaible, A. Scheuerlein, M. J. Dańko, J. Gampe, D. E. Martínez, J. W. Vaupel, Constant mortality and fertility over age in Hydra, Proc. Natl. Acad. Sci. U.S.A. 112 (51) (2015) 15701-15706. DOI:10.1073/pnas. 1521002112. 
[34] J. G. Ruby, M. Smith, R. Buffenstein, Naked mole-rat mortality rates defy gompertzian laws by not increasing with age, Elife 7 (2018) e31157. DOI :10.7554/eLife. 31157.

[35] D. Gems, L. Partridge, Genetics of longevity in model organisms: debates and paradigm shifts, Annu. Rev. Physiol. 75 (1) (2013) 621-644. DOI:10.1146/ annurev-physiol-030212-183712.

[36] C. Darwin, On the Origin of Species, 1859, Routledge, 2004 .

[37] T. W. Deacon, Incomplete Nature: How Mind Emerged from Matter, WW Norton \& Company, 2011.

[38] P. Nelson, J. Masel, Intercellular competition and the inevitability of multicellular aging, Proc. Natl. Acad. Sci. U.S.A. 114 (49) (2017) 12982-12987. DOI:10.1073/pnas. 1618854114.

[39] F. Kamangar, G. M. Dores, W. F. Anderson, Patterns of cancer incidence, mortality, and prevalence across five continents: defining priorities to reduce cancer disparities in different geographic regions of the world, J. Clin. Oncol. 24 (14) (2006) 2137-2150. DOI:10.1200/JC0.2005.05.2308.

[40] R. Buffenstein, Negligible senescence in the longest living rodent, the naked mole-rat: insights from a successfully aging species, J. Comp. Physiol. B Biochem. Syst. Environ. Physiol. 178 (4) (2008) 439-445. DOI:10.1007/ s00360-007-0237-5.

[41] M. A. Delaney, L. Nagy, M. J. Kinsel, P. M. Treuting, Spontaneous histologic lesions of the adult naked mole rat (Heterocephalus glaber): a retrospective survey of lesions in a zoo population, Vet. Pathol. 50 (4) (2013) 607-621. DOI : $10.1177 / 0300985812471543$.

[42] A. I. Rozhok, J. DeGregori, The evolution of lifespan and age-dependent cancer risk, Trends in Cancer 2 (10) (2016) 552-560. DOI: 10.1016/j.trecan.2016.09.004.

[43] P. Armitage, R. Doll, The age distribution of cancer and a multi-stage theory of carcinogenesis, Br. J. Cancer 8 (1) (1954) 1-12. DOI:10.1038/bjc.1954.1.

[44] T. W. Deacon, A. Srivastava, J. A. Bacigalupi, The transition from constraint to regulation at the origin of life, Front. Biosci. 19 (2014) 945-957. DOI:10.2741/4259.

[45] D. Karolchik, A. S. Hinrichs, T. S. Furey, K. M. Roskin, C. W. Sugnet, D. Haussler, W. J. Kent, The UCSC Table Browser data retrieval tool, Nucleic Acids Res. 32 (Database issue) (2004) D493-D496. DOI:10.1093/nar/ gkh103.

[46] W. J. Kent, A. S. Zweig, G. Barber, A. S. Hinrichs, D. Karolchik, BigWig and BigBed: enabling browsing of large distributed datasets, Bioinformatics 26 (17) (2010) 2204-2207. DOI:10.1093/bioinformatics/btq351.

[47] S. Neph, M. S. Kuehn, A. P. Reynolds, E. Haugen, R. E. Thurman, A. K. Johnson, E. Rynes, M. T. Maurano, J. Vierstra, S. Thomas, R. Sandstrom, R. Humbert, J. A. Stamatoyannopoulos, BEDOPS: high-performance genomic feature operations, Bioinformatics 28 (14) (2012) 1919-1920. DOI:10.1093/bioinformatics/bts277.
[48] A. R. Quinlan, I. M. Hall, BEDTools: a flexible suite of utilities for comparing genomic features, Bioinformatics 26 (6) (2010) 841-842. DOI:10.1093/bioinformatics/ btg033.

[49] C. E. Shannon, A mathematical theory of communication, Bell Syst. Tech. J. 27 (3) (1948) 379-423. DOI:10.1002/j. 1538-7305.1948. tb01338.x.

[50] W. McGill, Multivariate information transmission, Trans. IRE Prof. Gr. Inf. Theory 4 (4) (1954) 93-111. DOI:10. 1109/tit.1954.1057469.

[51] A. J. Bell, The co-information lattice, in: Proceedings of the Fifth International Workshop on Independent Component Analysis and Blind Signal Separation: ICA, Vol. 2003, 2003.

[52] W. H. Press, S. A. Teukolsky, W. T. Vetterling, B. P. Flannery, Numerical Recipes in C: The Art of Scientific Computing, Cambridge Univ. Press, 1992.

[53] D. Chicharro, S. Panzeri, Synergy and redundancy in dual decompositions of mutual information gain and information loss, Entropy 19 (71) (2017) 1-29. DOI: 10.3390/e19020071.

[54] J. Kruschke, Doing Bayesian Data Analysis: A Tutorial with R, JAGS, and Stan, Academic Press, 2014.

[55] G. P. Wagner, K. Kin, V. J. Lynch, Measurement of mRNA abundance using RNA-seq data: RPKM measure is inconsistent among samples, Theory Biosci. 131 (4) (2012) 281-285. DOI : 10.1007/s12064-012-0162-3.

[56] R. E. Walpole, Essentials of Probability \& Statistics for Engineers \& Scientists, Pearson, 2013.

[57] F. Kenney, E. S. Keeping, Mathematics of Statistics: Part Two, D. Van Nostrand Company, Inc., 1951.

[58] H. B. Mann, D. R. Whitney, On a test of whether one of two random variables is stochastically larger than the other, Ann. Math. Stat. 18 (1) (1947) 50-60. DOI:10.1214/ aoms/1177730491.

[59] Y. Benjamini, D. Yekutieli, The control of the false discovery rate in multiple testing under dependency, Ann. Stat. (2001) 1165-1188. Available from: http://www. jstor. org/stable/2674075.

[60] Y. Benjamini, Y. Hochberg, Controlling the false discovery rate: a practical and powerful approach to multiple testing, J. R. Stat. Soc. Ser. B 50 (1) (1995) 289-300. Available from: http://www. jstor.org/stable/2346101.

[61] R Core Team, R: A Language and Environment for Statistical Computing (2017). Available from: https: //www.r-project.org/.

[62] P. E. Meyer, infotheo: Information-Theoretic Measures (2014). Available from: https://cran.r-project.org/ package=infotheo.

[63] L. Paninski, Estimation of entropy and mutual information, Neural Comput. 15 (6) (2003) 1191-1253. DOI : 10.1162/089976603321780272.

[64] G. A. Miller, Note on the bias of information estimates, in: Information Theory in Psychology: Problems and Methods II-B, The Free Press of Glencoe, 1955, pp. 95-100. 